\title{
Limit behavior of mass critical Hartree minimization problems with steep potential wells
}

Yujin Guo, Yong Luo, and Zhi-Qiang Wang

Citation: Journal of Mathematical Physics 59, 061504 (2018); doi: 10.1063/1.5025730

View online: https://doi.org/10.1063/1.5025730

View Table of Contents: http://aip.scitation.org/toc/jmp/59/6

Published by the American Institute of Physics

\section{Articles you may be interested in}

Existence of solutions for a generalized Yang-Mills-Higgs theory

Journal of Mathematical Physics 59, 061501 (2018); 10.1063/1.5004044

Global solutions and finite time blow-up for fourth order nonlinear damped wave equation Journal of Mathematical Physics 59, 061503 (2018); 10.1063/1.5006728

Nonlocal nonlinear Schrödinger equations and their soliton solutions Journal of Mathematical Physics 59, 051501 (2018); 10.1063/1.4997835

Threshold behavior and uniqueness of ground states for mass critical inhomogeneous Schrödinger equations

Journal of Mathematical Physics 59, 011503 (2018); 10.1063/1.5008924

Continuous quasiperiodic Schrödinger operators with Gordon type potentials Journal of Mathematical Physics 59, 063501 (2018); 10.1063/1.5005076

Schwarzian derivative and Ermakov equation on a time scale Journal of Mathematical Physics 59, 061502 (2018); 10.1063/1.5022325

\section{PHYSICS TODAY}

WHITEPAPERS
MANAGER'S GUIDE

Accelerate R\&D with

Multiphysics Simulation
READ NOW

PRESENTED BY

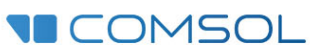




\title{
Limit behavior of mass critical Hartree minimization problems with steep potential wells
}

\author{
Yujin Guo, ${ }^{1, a)}$ Yong Luo, ${ }^{2, b)}$ and Zhi-Qiang Wang ${ }^{3, c)}$ \\ ${ }^{1}$ Wuhan Institute of Physics and Mathematics, Chinese Academy of Sciences, P.O. Box 71010, \\ Wuhan 430071, People's Republic of China \\ ${ }^{2}$ University of Chinese Academy of Sciences, Beijing 100190, People's Republic of China \\ and Wuhan Institute of Physics and Mathematics, Chinese Academy of Sciences, \\ P.O. Box 71010, Wuhan 430071, People's Republic of China \\ ${ }^{3}$ Center for Applied Mathematics, Tianjin University, Tianjin 300072, People's Republic \\ of China and Department of Mathematics and Statistics, Utah State University, Logan, \\ Utah 84322, USA
}

(Received 12 February 2018; accepted 21 May 2018; published online 7 June 2018)

We consider minimizers of the following mass critical Hartree minimization problem: $e_{\lambda}(N):=\inf _{\left\{u \in H^{1}\left(\mathbb{R}^{d}\right),\|u\|_{2}^{2}=N\right\}} E_{\lambda}(u)$, where $d \geq 3, \lambda>0$, and the Hartree energy functional $E_{\lambda}(u)$ is defined by $E_{\lambda}(u):=\int_{\mathbb{R}^{d}}|\nabla u(x)|^{2} d x+\lambda \int_{\mathbb{R}^{d}} g(x) u^{2}(x) d x$ $-\frac{1}{2} \int_{\mathbb{R}^{d}} \int_{\mathbb{R}^{d}} \frac{u^{2}(x) u^{2}(y)}{|x-y|^{2}} d x d y$. Here the steep potential $g(x)$ satisfies $0=g(0)=\inf _{\mathbb{R}^{d}} g(x)$ $\leq g(x) \leq 1$ and $1-g(x) \in L^{\frac{d}{2}}\left(\mathbb{R}^{d}\right)$. We prove that there exists a constant $N^{*}>0$, independent of $\lambda g(x)$, such that if $N \geq N^{*}$, then $e_{\lambda}(N)$ does not admit minimizers for any $\lambda>0$; if $0<N<N^{*}$, then there exists a constant $\lambda^{*}(N)>0$ such that $e_{\lambda}(N)$ admits minimizers for any $\lambda>\lambda^{*}(N)$ and $e_{\lambda}(N)$ does not admit minimizers for $0<\lambda<\lambda^{*}(N)$. For any given $0<N<N^{*}$, the limit behavior of positive minimizers for $e_{\lambda}(N)$ is also studied as $\lambda \rightarrow \infty$, where the mass concentrates at the bottom of $g(x)$. Published by AIP Publishing. https://doi.org/10.1063/1.5025730

\section{INTRODUCTION}

In the 1980s, Lions et al. analyzed in Refs. 7 and 19 the following Hartree minimization problem:

$$
\begin{gathered}
\inf \left\{\int_{\mathbb{R}^{d}}\left(|\nabla u(x)|^{2}+V(x) u^{2}(x)\right) d x-\frac{1}{2} \int_{\mathbb{R}^{d}} \int_{\mathbb{R}^{d}} \frac{u^{2}(x) u^{2}(y)}{|x-y|} d y d x,\right. \\
\left.u \in H^{1}\left(\mathbb{R}^{d}\right), \quad \int_{\mathbb{R}^{d}} u^{2} d x=N>0\right\},
\end{gathered}
$$

where $d \geq 3$ and $V(x) \geq 0$ is an external potential. The problem (1.1) arises in quantum mechanics; see Refs. 16 and 19 for some discussions on the relevance of this problem to physics. Especially, the existence of positive minimizers for (1.1) was discussed in Refs. 7 and 19 by developing and applying the celebrated concentration-compactness principle. After these pioneering studies, the problem (1.1) was studied widely over the past few decades. We remark that the problem (1.1) is essentially a mass subcritical problem and some analysis of (1.1) can be extended naturally to the general case where the term $\frac{1}{|x-y|}$ in (1.1) is replaced by $\frac{1}{|x-y|^{s}}$ for all $0<s<2$.

On the other hand, the mass critical minimization problem, arising in Bose-Einstein condensates (BEC) in $\mathbb{R}^{2}$, was analyzed recently in Refs. 8 and 12-14 and references therein, where the authors focused on the class where $V(x)$ satisfies

$$
0 \leq V(x) \in L_{\mathrm{loc}}^{\infty}\left(\mathbb{R}^{2}\right) \text { and } \lim _{|x| \rightarrow \infty} V(x)=\infty .
$$

\footnotetext{
a)Email: yjguo@wipm.ac.cn.

b) Email: luoyong.wipm@outlook.com.

c)Email: zhi-qiang.wang@usu.edu.
} 
Under the assumption (1.2), the mass critical case of (1.1), where the term $\frac{1}{|x-y|}$ is replaced by $\frac{1}{|x-y|^{2}}$, was studied in Ref. 10 and somewhere else. Stimulated by above facts, in this paper, we are interested in the mass critical case of (1.1) with a steep potential well $V(x) \in L^{\infty}\left(\mathbb{R}^{d}\right)$. We remark that the steep potential wells were considered in Refs. 1 and 2 in the setting of nonlinear Schrödinger equations without constraints. And here, we consider normalized solutions subject to $L^{2}$ norm constraints which are more in line of concern with stability issues of standing waves.

More precisely, in this paper, we investigate the following mass critical minimization problem:

$$
e_{\lambda}(N):=\inf _{u \in H^{1}\left(\mathbb{R}^{d}\right),\|u\|_{2}^{2}=N} E_{\lambda}(u), d \geq 3,
$$

where the Hartree energy functional $E_{\lambda}(u)$ satisfies

$$
E_{\lambda}(u):=\int_{\mathbb{R}^{d}}|\nabla u(x)|^{2} d x+\lambda \int_{\mathbb{R}^{d}} g(x) u^{2}(x) d x-\frac{1}{2} \int_{\mathbb{R}^{d}} \int_{\mathbb{R}^{d}} \frac{u^{2}(x) u^{2}(y)}{|x-y|^{2}} d x d y, \lambda>0 .
$$

Here we consider the steep potential $g(x) \in C_{l o c}^{\alpha}\left(\mathbb{R}^{d}\right)$ with $\alpha \in(0,1)$ satisfying

$\left(\mathrm{M}_{1}\right) .0=g(0)=\inf _{x \in \mathbb{R}^{d}} g(x) \leq g(x) \leq 1,1-g(x) \in L^{\frac{d}{2}}\left(\mathbb{R}^{d}\right)$, where $0 \in \mathbb{R}^{d}$ is the unique global minimum point of $g(x)$ in $\mathbb{R}^{d}$.

The main purposes of this paper are to classify the existence and nonexistence of minimizers for $e_{\lambda}(N)$, based on which we shall investigate the limit behavior of minimizers as $\lambda \rightarrow \infty$. Even though there are existing papers as mentioned above of studying mass critical minimization problems, as far as we know, this paper might be the first work of studying mass critical minimization problems under the steep potential $g(x)$ satisfying $\left(M_{1}\right)$. More importantly, our analysis shows that there appear new and interesting phenomena on the existence and nonexistence of minimizers for $e_{\lambda}(N)$, and the limit behavior of minimizers as $\lambda \rightarrow \infty$ presents new and challenging difficulties, for which one needs to investigate new analytic approaches.

Related to the minimization problem $e_{\lambda}(N)$, to state our main results, we now introduce the following nonlocal Hartree equation:

$$
-\triangle u+u-\left(\int_{\mathbb{R}^{d}} \frac{u^{2}(y)}{|x-y|^{2}} d y\right) u=0 \text { in } \mathbb{R}^{d}, 0<u \in H^{1}\left(\mathbb{R}^{d}\right) \text { and } d \geq 3 .
$$

We define the energy functional of (1.5) by

$$
I(u):=\frac{1}{2} \int_{\mathbb{R}^{d}}|\nabla u(x)|^{2}+u^{2}(x) d x-\frac{1}{4} \int_{\mathbb{R}^{d}} \int_{\mathbb{R}^{d}} \frac{u^{2}(x) u^{2}(y)}{|x-y|^{2}} d x d y, u \in H^{1}\left(\mathbb{R}^{d}\right) .
$$

Consider

$$
S:=\left\{u(x) \in H^{1}\left(\mathbb{R}^{d}\right): u(x) \text { is a positive solution of }(1.5)\right\}
$$

and

$$
G:=\{u(x) \in S: I(u) \leq I(v), \text { for all } v \in S\} .
$$

We then say that $0<u \in H^{1}\left(\mathbb{R}^{d}\right)$ is a ground state of (1.5) if $u \in G$. Note from Refs. 21 and 23 that (1.5) admits ground states $Q>0$, which must be radially symmetric and admit the following exponential decay:

$$
Q(|x|),|\nabla Q(|x|)|=O\left(e^{-\mu|x|}\right) \text { as }|x| \rightarrow \infty, \text { where } \mu>0 .
$$

We further remark from Ref. 23, Proposition 3.1 that any solution $u$ of (1.5) satisfies the following Pohozaev-type identity:

$$
\frac{d-2}{2} \int_{\mathbb{R}^{d}}|\nabla u(x)|^{2} d x+\frac{d}{2} \int_{\mathbb{R}^{d}}|u(x)|^{2} d x=\frac{d-1}{2} \int_{\mathbb{R}^{d}} \int_{\mathbb{R}^{d}} \frac{u^{2}(x) u^{2}(y)}{|x-y|^{2}} d x d y .
$$

Following this identity, one can obtain that any solution $u(x)$ of (1.5) satisfies

$$
\int_{\mathbb{R}^{d}}|\nabla u(x)|^{2} d x=\int_{\mathbb{R}^{d}} u^{2}(x) d x=\frac{1}{2} \int_{\mathbb{R}^{d}} \frac{u^{2}(x) u^{2}(y)}{|x-y|^{2}} d x d y .
$$


Combining (1.6) and (1.11), we know that all ground states $Q>0$ of (1.5) share the same $L^{2}$-norm, i.e.,

$$
N^{*}=\int_{\mathbb{R}^{d}} Q^{2}(x) d x, \forall Q \in G .
$$

We finally note from Ref. 4 the following Gagliardo-Nirenberg inequality:

$$
\int_{\mathbb{R}^{d}} \int_{\mathbb{R}^{d}} \frac{u^{2}(x) u^{2}(y)}{|x-y|^{2}} d x d y \leq \frac{2}{N^{*}} \int_{\mathbb{R}^{d}}|\nabla u(x)|^{2} d x \int_{\mathbb{R}^{d}} u^{2}(x) d x, u \in H^{1}\left(\mathbb{R}^{d}\right),
$$

where the identity is attained at any ground state $Q=Q(|x|)$ of (1.5). Denote $S_{d}>0$ as the optimal constant of the following Sobolev inequality:

$$
\left(\int_{\mathbb{R}^{d}}|u|^{2^{*}} d x\right)^{\frac{2}{2^{*}}} \leq S_{d}^{-1} \int_{\mathbb{R}^{d}}|\nabla u|^{2} d x, u \in H^{1}\left(\mathbb{R}^{d}\right),
$$

where $2^{*}=\frac{2 d}{d-2}$. Following above notations, our first main result of this paper is concerned with the existence and nonexistence of minimizers in terms of parameters $N$ and $\lambda$.

Theorem 1.1. Suppose $g(x)$ satisfies the assumption $\left(\mathrm{M}_{1}\right)$ and let $N^{*}:=\|Q\|_{2}^{2}>0$ be defined by (1.12). Then we have

(i). If $N \geq N^{*}$, there is no minimizer of $e_{\lambda}(N)$ for any $\lambda>0$.

(ii). If $0<N<N^{*}$, then there exists a positive constant $\lambda^{*}(N)$ satisfying

$$
\frac{S_{d}\left(N^{*}-N\right)}{N^{*}\|1-g\|_{L^{\frac{d}{2}}}} \leq \lambda^{*}(N) \leq \frac{N^{*}-N}{\int_{\mathbb{R}^{d}}(1-g) Q^{2} d x}
$$

such that

(a) If $\lambda>\lambda^{*}(N)$, then there exists at least one minimizer for $e_{\lambda}(N)$.

(b) If $0<\lambda<\lambda^{*}(N)$, then there is no minimizer for $e_{\lambda}(N)$.

The existence and non-existence of minimizers in Theorem 1.1 can be illustrated explicitly by Fig. 1. Theorem 1.1 reveals the following interesting phenomenon: the depth $\lambda$ of the steep potential well $g(x)$ must be large enough to keep the mass of minimizing sequences in a compact domain of $\mathbb{R}^{d}$, which ensures the existence of minimizers for $e_{\lambda}(N)$. We shall prove that the finite constant $\lambda^{*}(N)$ in Theorem 1.1 can be characterized by

$$
\lambda^{*}(N)=\inf _{u \in H^{1}\left(\mathbb{R}^{d}\right),\|u\|_{2}^{2}=N} \frac{\int_{\mathbb{R}^{d}}|\nabla u|^{2} d x-\frac{1}{2} \int_{\mathbb{R}^{d}} \int_{\mathbb{R}^{d}} \frac{u^{2}(x) u^{2}(y)}{|x-y|^{2}} d x d y}{\int_{\mathbb{R}^{d}}(1-g) u^{2} d x},
$$

where the assumption $1-g(x) \in L^{\frac{d}{2}}\left(\mathbb{R}^{d}\right)$ is to guarantee that $\lambda^{*}(N)>0$. Indeed, if $g(x)$ satisfies $\liminf _{|x| \rightarrow \infty}(1-g(x))|x|^{2}=+\infty$, then one can get from (1.15) by scaling that $\lambda^{*}(N) \equiv 0$, which further implies the existence of minimizers for $e_{\lambda}(N)$ at any $0<N<N^{*}$ and $\lambda>0$.

Suppose $u_{\lambda}$ is a real-valued minimizer of $e_{\lambda}(N)$, then $u_{\lambda}$ satisfies the following Euler-Lagrange equation:

$$
-\Delta u_{\lambda}+\lambda g(x) u_{\lambda}=\mu_{\lambda} u_{\lambda}+\left(\int_{\mathbb{R}^{d}} \frac{u_{\lambda}^{2}(y)}{|x-y|^{2}} d y\right) u_{\lambda} \text { in } \mathbb{R}^{d},
$$

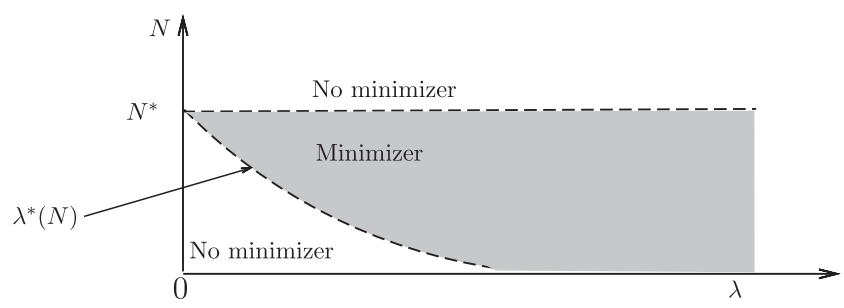

FIG. 1. Existence of minimizers for $e_{\lambda}(N)$ at different value $(\lambda, N)$. 
where $\mu_{\lambda} \in \mathbb{R}$ is the associated Lagrange multiplier. Since the energy functional $E_{\lambda}(\cdot)$ is even, we now obtain from Ref. 17, Theorem 6.17 that $E_{\lambda}\left(\left|u_{\lambda}\right|\right)=E_{\lambda}\left(u_{\lambda}\right)$, which implies that $\left|u_{\lambda}\right|$ is also a minimizer of $e_{\lambda}(N)$. By the strong maximum principle, one can further derive from the corresponding Euler-Lagrange equation of $\left|u_{\lambda}\right|$ to obtain that $\left|u_{\lambda}\right|>0$ holds in $\mathbb{R}^{d}$. Therefore, $u_{\lambda}$ must be either positive or negative. Without loss of generality, in the following, we only consider positive minimizers $u_{\lambda}>0$ of $e_{\lambda}(N)$. We shall focus on the limit behavior of positive minimizers for $e_{\lambda}(N)$ as $\lambda \rightarrow \infty$, where $0<N<N^{*}$ is arbitrary and $g(x)$ satisfies $\left(M_{1}\right)$ and

$\left(\mathrm{M}_{2}\right), g(x) \in C_{l o c}^{\alpha}\left(\mathbb{R}^{d}\right)$ for some $\alpha \in(0,1)$, and $g(x)=|x|^{p}[1+o(1)]$ as $|x| \rightarrow 0$, where $p>0$.

Under the above assumptions, our second main result of this paper can be stated as the following theorem.

Theorem 1.2. Suppose $g(x)$ satisfies $\left(\mathrm{M}_{1}\right)$ and $\left(\mathrm{M}_{2}\right)$ for $p>0$ and $N \in\left(0, N^{*}\right)$ is arbitrary. Let $u_{\lambda_{k}}$ be a positive minimizer of $e_{\lambda_{k}}(N)$, where $\lambda_{k} \nearrow \infty$ as $k \rightarrow \infty$. Then there exists a subsequence of $\left\{\lambda_{k}\right\}$ (still denoted by $\left\{\lambda_{k}\right\}$ ) such that

$$
\lim _{k \rightarrow \infty} \lambda_{k}^{-\frac{d}{2(2+p)}} u_{\lambda_{k}}\left(\lambda_{k}^{-\frac{1}{2+p}} x+x_{\lambda_{k}}\right)=w_{0}(x) \text { in } H^{1}\left(\mathbb{R}^{d}\right),
$$

where $x_{\lambda_{k}} \in \mathbb{R}^{d}$ is a maximal point of $u_{\lambda_{k}}$, which satisfies $\lim _{k \rightarrow \infty} \lambda_{k}^{\frac{1}{2+p}} x_{\lambda_{k}}=0$, and $w_{0}>0$ is a ground state of the following equation:

$$
-\triangle w_{0}+|x|^{p} w_{0}=\mu w_{0}+\left(\int_{\mathbb{R}^{d}} \frac{w_{0}^{2}(y)}{|x-y|^{2}} d y\right) w_{0} \text { in } \mathbb{R}^{d}, \quad w_{0} \in H^{1}\left(\mathbb{R}^{d}\right) .
$$

Here $\mu=\mu(N) \in \mathbb{R}$ is determined by the constraint $\int_{\mathbb{R}^{d}} w_{0}^{2}=N$.

The proof of Theorem 1.2 shows that $w_{0}>0$ in (1.17) is essentially a positive minimizer of the following minimization problem:

$$
e_{\infty}(N)=\inf _{\left\{u \in \mathcal{H},\|u\|_{2}^{2}=N\right\}} E_{\infty}(u),
$$

where the space $\mathcal{H}$ is defined by

$$
\mathcal{H}=\left\{u \in H^{1}\left(\mathbb{R}^{d}\right): \int_{\mathbb{R}^{d}}|x|^{p} u^{2} d x<\infty\right\}, p>0
$$

and the energy functional $E_{\infty}(u)$ is of the form

$$
E_{\infty}(u)=\int_{\mathbb{R}^{d}}|\nabla u|^{2} d x+\int_{\mathbb{R}^{d}}|x|^{p} u^{2} d x-\frac{1}{2} \int_{\mathbb{R}^{d}} \int_{\mathbb{R}^{d}} \frac{u^{2}(x) u^{2}(y)}{|x-y|^{2}} d x d y, \quad u \in \mathcal{H} .
$$

The arguments of Refs. 10 and 12 give that $e_{\infty}(N)$ admits positive minimizers $w_{0}>0$ if and only if $N \in\left(0, N^{*}\right)$, where $N^{*}=\|Q\|_{2}^{2}$ and $Q>0$ is still a ground state of (1.5). As illustrated in Proposition 3.1 , any positive minimizer $w_{0}>0$ of $e_{\infty}(N)$ must be radially symmetric and is strictly decreasing in $|x|$. Moreover, $e_{\infty}(N)$ admits a unique positive minimizer, for the cases where either $N>0$ is small enough (cf. Ref. 14, Theorem 1.1) or $N$ is close enough to $N^{*}$ for $p \geq 2$ and $d=4$ (cf. Ref. 20).

In spite of above facts, we cannot obtain enough information, including the uniqueness and nondegeneracy, on positive minimizers of $e_{\infty}(N)$ for all cases of $0<N<N^{*}$ and $d \geq 3$. This leads to some new and challenging difficulties in the proof of Theorem 1.2, comparing with those appeared in Refs. 8, 10, and 12-14 and references therein. Actually, the existing methods in the above mentioned papers are only applicable to the case where $N<N^{*}$ is close sufficiently to $N^{*}$. To prove Theorem 1.2 for the general case where $0<N<N^{*}$ is arbitrary, we shall establish Lemmas 3.4 and 3.5 on different types of estimates. Moreover, we need to seek for a different approach of deriving the lower bound of $e_{\lambda}(N)$, for which we shall borrow some ideas from Ref. 3 .

This paper is organized as follows. Section II is devoted to the proof of Theorem 1.1 on the existence and nonexistence of minimizers. In Sec. III, we first address some a priori estimates of positive minimizers for $e_{\lambda}(N)$ as $\lambda \rightarrow \infty$, after which we shall complete the proof of Theorem 1.2. 


\section{EXISTENCE OF MINIMIZERS}

In this section, we shall complete the proof of Theorem 1.1 under the assumption $\left(M_{1}\right)$. For convenience, we first define a new minimization problem

$$
\hat{e}_{\lambda}(N):=\inf _{\left\{u \in H^{1}\left(\mathbb{R}^{d}\right),\|u\|_{2}^{2}=N\right\}} \hat{E}_{\lambda}(u), d \geq 3,
$$

where the energy functional $\hat{E}_{\lambda}(u)$ satisfies for any $\lambda>0$,

$$
\hat{E}_{\lambda}(u):=\int_{\mathbb{R}^{d}}|\nabla u(x)|^{2} d x-\lambda \int_{\mathbb{R}^{d}}[1-g(x)] u^{2}(x) d x-\frac{1}{2} \int_{\mathbb{R}^{d}} \int_{\mathbb{R}^{d}} \frac{u^{2}(x) u^{2}(y)}{|x-y|^{2}} d x d y
$$

and $0 \leq g(x) \leq 1$ satisfies the assumption $\left(M_{1}\right)$. Since $\hat{E}_{\lambda}(u)=E_{\lambda}(u)-\lambda N$, to prove Theorem 1.1 it suffices to address the existence and nonexistence of minimizers for $\hat{e}_{\lambda}(N)$, instead of $e_{\lambda}(N)$. We start with the following lemma.

Lemma 2.1. Suppose $g(x)$ satisfies the assumption $\left(\mathrm{M}_{1}\right)$ and let $N^{*}:=\|Q\|_{2}^{2}>0$ be defined by (1.12). Then

1. If $N \geq N^{*}$, then there is no minimizer of (2.1) for any $\lambda>0$.

2. If $0<N<N^{*}$, then $\hat{e}_{\lambda}(N) \leq 0$ for any $\lambda>0$.

Proof. (i) We first consider the case where $N>N^{*}$ and $\lambda>0$ are arbitrary. Set

$$
u_{\theta}(x)=\left(\frac{N}{N^{*}} \theta^{d}\right)^{\frac{1}{2}} Q(\theta x), \theta>0,
$$

where $Q>0$ is a ground state of (1.5). By applying the Gagliardo-Nirenberg inequality (1.13), we then have for any $N>N^{*}$ and $\lambda>0$,

$$
\begin{aligned}
\hat{e}_{\lambda}(N) & \leq \hat{E}_{\lambda}\left(u_{\theta}(x)\right) \\
& \leq \int_{\mathbb{R}^{d}} \frac{N}{N^{*}} \theta^{d}|\nabla Q(\theta x)|^{2} \theta^{2} d x-\frac{N^{2}}{2 N^{* 2}} \int_{\mathbb{R}^{d}} \int_{\mathbb{R}^{d}} \frac{\theta^{2 d} Q^{2}(\theta x) Q^{2}(\theta y)}{|x-y|^{2}} d x d y \\
& =N\left(1-\frac{N}{N^{*}}\right) \theta^{2} \rightarrow-\infty \text { as } \theta \rightarrow \infty,
\end{aligned}
$$

due to (1.11), which thus implies that $\hat{e}_{\lambda}(N)=-\infty$ in this case. This shows the nonexistence of minimizers for $\hat{e}_{\lambda}(N)$, where $N>N^{*}$ and $\lambda>0$.

Next, if $N=N^{*}$, we follow again the Gagliardo-Nirenberg inequality (1.13) to derive that for any $\lambda>0$,

$$
\hat{E}_{\lambda}(u) \geq-\lambda \int_{\mathbb{R}^{d}}(1-g) u^{2} d x \geq-\lambda N^{*} \text {, i.e., } \hat{e}_{\lambda}\left(N^{*}\right) \geq-\lambda N^{*}
$$

On the other hand, choose a cutoff function $0 \leq \varphi \in C_{0}^{\infty}\left(\mathbb{R}^{d}\right)$ such that $\varphi(x)=1$ for $|x| \leq 1$ and $\varphi(x)=0$ for $|x| \geq 2$. For $\tau>0$, let

$$
u_{\tau}(x)=A_{\tau} \frac{\tau^{\frac{d}{2}}}{\|Q\|_{2}} \varphi(x) Q(\tau x)
$$

and $A_{\tau}>0$ is chosen so that $\int_{\mathbb{R}^{d}} u_{\tau}^{2}(x) d x=N^{*}$. Using (1.9), we have for some $C>0$,

$$
N^{*} \leq A_{\tau}^{2} \leq N^{*}+O\left(e^{-C \tau}\right) \text { as } \tau \rightarrow \infty .
$$

By (1.9), one can calculate that

$$
\int_{\mathbb{R}^{d}}\left|\nabla u_{\tau}\right|^{2} d x \leq \frac{A_{\tau}^{2} \tau^{2}}{\|Q\|_{2}^{2}} \int_{\mathbb{R}^{d}}|\nabla Q|^{2} d x+O\left(\tau^{2} e^{-C \tau}\right) \text { as } \tau \rightarrow \infty .
$$


As for the nonlocal term, we have

$$
\begin{aligned}
& \int_{\mathbb{R}^{d}} \int_{\mathbb{R}^{d}} \frac{u_{\tau}^{2}(x) u_{\tau}^{2}(y)}{|x-y|^{2}} d x d y \\
= & \frac{A_{\tau}^{4} \tau^{2}}{\|Q\|_{2}^{4}} \int_{\mathbb{R}^{d}} \int_{\mathbb{R}^{d}} \frac{\varphi^{2}\left(\frac{x}{\tau}\right) \varphi^{2}\left(\frac{y}{\tau}\right) Q^{2}(x) Q^{2}(y)}{|x-y|^{2}} d x d y \\
= & \frac{A_{\tau}^{4} \tau^{2}}{\|Q\|_{2}^{4}} \int_{\mathbb{R}^{d}} \int_{\mathbb{R}^{d}} \frac{Q^{2}(x) Q^{2}(y)}{|x-y|^{2}} d x d y+O\left(\tau^{2} e^{-C \tau}\right) \text { as } \tau \rightarrow \infty .
\end{aligned}
$$

By (2.6) and (2.7), we obtain from (1.11) that

$$
\begin{aligned}
\hat{e}_{\lambda}\left(N^{*}\right) \leq \hat{E}_{\lambda}\left(u_{\tau}(x)\right) & \leq-\lambda \int_{\mathbb{R}^{d}}(1-g) u_{\tau}^{2}(x) d x+O\left(\tau^{2} e^{-C \tau}\right) \\
& =\frac{A_{\tau}^{2}}{\|Q\|_{2}^{2}} \lambda \int_{\mathbb{R}^{d}} g\left(\frac{x}{\tau}\right) \varphi^{2}\left(\frac{x}{\tau}\right) Q^{2}(x) d x-\lambda N^{*}+O\left(\tau^{2} e^{-C \tau}\right) \\
& =-\lambda N^{*}+o(1) \text { as } \tau \rightarrow \infty .
\end{aligned}
$$

We then conclude from (2.4) and (2.8) that $\hat{e}_{\lambda}\left(N^{*}\right)=-\lambda N^{*}$. If there were a minimizer $u$, we would have

$$
\int_{\mathbb{R}^{d}} g(x) u^{2}(x) d x=0=\inf _{x \in \mathbb{R}^{d}} g(x)
$$

and

$$
\int_{\mathbb{R}^{d}}|\nabla u(x)|^{2} d x=\frac{2}{N^{*}} \int_{\mathbb{R}^{d}} \int_{\mathbb{R}^{d}} \frac{u^{2}(x) u^{2}(y)}{|x-y|^{2}} d x d y,
$$

which is a contradiction since $u$ would have compact support by the first equality, while it has to be equal to $Q(x)$ (up to a scaling) in view of the second equality. Hence, we also have the nonexistence of minimizers for $\hat{e}_{\lambda}(N)$ in the case where $N=N^{*}$ and $\lambda>0$.

(ii) Using the same trial function (2.3), one can derive that

$$
\hat{e}_{\lambda}(N) \leq \hat{E}_{\lambda}\left(u_{\theta}(x)\right)=\hat{E}_{\lambda}\left(\left(\frac{N}{N^{*}} \theta^{d}\right)^{\frac{1}{2}} Q(\theta x)\right) \leq N \theta^{2}\left(1-\frac{N}{N^{*}}\right) .
$$

Following above estimates by taking $\theta \rightarrow 0$, we thus conclude that $\hat{e}_{\lambda}(N) \leq 0$ holds for any $0<N$ $<N^{*}$ and $\lambda>0$, and the proof is therefore complete.

We next employ the celebrated concentration-compactness lemma (cf. Ref. 19) to prove the following existence of minimizers.

Lemma 2.2. If $\hat{e}_{\lambda}(N)<0$ holds for some $0<N<N^{*}$ and $\lambda>0$, then there exist minimizers for (2.1).

Proof. For any $0<N<N^{*}$ and $\lambda>0$, we have

$$
\begin{aligned}
\hat{E}_{\lambda}(u) & =\int_{\mathbb{R}^{d}}|\nabla u(x)|^{2} d x-\lambda \int_{\mathbb{R}^{d}}[1-g(x)] u^{2}(x) d x-\frac{1}{2} \int_{\mathbb{R}^{d}} \int_{\mathbb{R}^{d}} \frac{u^{2}(x) u^{2}(y)}{|x-y|^{2}} d x d y \\
& \geq\left(1-\frac{N}{N^{*}}\right) \int_{\mathbb{R}^{d}}|\nabla u(x)|^{2} d x-\lambda N, \quad \forall u \in H^{1}\left(\mathbb{R}^{d}\right) .
\end{aligned}
$$

This implies that any minimizing sequence $\left\{u_{n}\right\}$ of $\hat{e}_{\lambda}(N)$ is bounded uniformly in $H^{1}\left(\mathbb{R}^{d}\right)$ and $\left\|u_{n}\right\|_{2}^{2}=N$. It then follows from Ref. 19, Lemma III.1 that there exists a subsequence $\left\{u_{n_{k}}\right\}$ of $\left\{u_{n}\right\}$ such that either the compactness or the dichotomy or the vanishing occurs for the subsequence $\left\{u_{n_{k}}\right\}$.

We first prove that the vanishing

$$
\limsup _{k \rightarrow \infty, y \in \mathbb{R}^{d}} \int_{B_{R(y)}} u_{n_{k}}^{2}(x) d x=0, \quad \forall R<\infty
$$


cannot occur. On the contrary, suppose that (2.10) is true. Then, we infer from Ref. 24, Lemma 1.21 that

$$
u_{n_{k}} \stackrel{k}{\rightarrow} 0 \text { in } L^{q}\left(\mathbb{R}^{d}\right), \text { where } 2<q<2^{*} .
$$

Following (2.11), since $g(x)$ satisfies $\left(\mathrm{M}_{1}\right)$, we obtain that

$$
\int_{\mathbb{R}^{d}}[1-g(x)] u_{n_{k}}^{2} d x \leq \int_{\mathbb{R}^{d}}[1-g(x)]^{\frac{1}{2}} u_{n_{k}}^{2} d x \leq\|1-g\|_{\frac{d}{2}}^{\frac{1}{2}}\left\|u_{n_{k}}\right\|_{\frac{2 d}{d-1}}^{2} \stackrel{k}{\rightarrow} 0 .
$$

Similarly, by the Hardy-Littlewood-Sobolev inequality (cf. Ref. 17, Theorem 4.3), we derive from (2.11) that

$$
\int_{\mathbb{R}^{d}} \int_{\mathbb{R}^{d}} \frac{u_{n_{k}}^{2}(x) u_{n_{k}}^{2}(y)}{|x-y|^{2}} d x d y \leq C\left\|u_{n_{k}}\right\|_{\frac{2 d}{d-1}}^{4} \rightarrow 0 \text { as } k \rightarrow \infty .
$$

Using (2.12) and (2.13), we conclude that $\hat{e}_{\lambda}(N)=0$ in view of Lemma 2.1 (2), which however contradicts the hypothesis that $\hat{e}_{\lambda}(N)<0$. This proves the claim $(2.10)$.

To rule out the dichotomy, we now claim that for any $N \in\left(0, N^{*}\right)$,

$$
\hat{e}_{\lambda}(\theta \alpha)<\theta \hat{e}_{\lambda}(\alpha), \forall \alpha \in(0, N), \forall \theta \in\left(1, \frac{N}{\alpha}\right] .
$$

Indeed, for any $\lambda>0$ and $0<N<N^{*}$, the estimate (2.9) implies that $\hat{e}_{\lambda}(\alpha)$ is bounded from below for any $\alpha \in(0, N)$. Let $\left\{v_{n}\right\} \subset H^{1}\left(\mathbb{R}^{d}\right)$ be a minimizing sequence of $\hat{e}_{\lambda}(\alpha)$ so that for any $n \in \mathbb{N}$, we have $\left\|v_{n}\right\|_{2}^{2}=\alpha$ and $\lim _{n \rightarrow \infty} \hat{E}_{\lambda}\left(v_{n}\right)=\hat{e}_{\lambda}(\alpha)$. It then follows from the estimate (2.9) that $v_{n}$ is bounded uniformly in $H^{1}\left(\mathbb{R}^{d}\right)$. Set $v_{\theta, n}:=\theta^{\frac{1}{2}} v_{n}(x)$ such that $\left\|v_{\theta, n}\right\|_{2}^{2}=\theta \alpha$. Since $\theta>1$, we obtain from (2.1) that

$$
\begin{aligned}
\hat{e}_{\lambda}(\theta \alpha) \leq & \lim _{n \rightarrow \infty} \hat{E}_{\lambda}\left(v_{\theta, n}\right) \\
= & \lim _{n \rightarrow \infty}\left\{\theta \int_{\mathbb{R}^{d}}\left|\nabla v_{n}(x)\right|^{2} d x-\theta \lambda \int_{\mathbb{R}^{d}}[1-g(x)] v_{n}^{2}(x) d x\right. \\
& \left.-\frac{1}{2} \theta^{2} \int_{\mathbb{R}^{d}} \int_{\mathbb{R}^{d}} \frac{v_{n}^{2}(x) v_{n}^{2}(y)}{|x-y|^{2}} d x d y\right\} \\
= & \theta \hat{e}_{\lambda}(\alpha)+\frac{\theta(1-\theta)}{2} \lim _{n \rightarrow \infty} \int_{\mathbb{R}^{d}} \int_{\mathbb{R}^{d}} \frac{v_{n}^{2}(x) v_{n}^{2}(y)}{|x-y|^{2}} d x d y .
\end{aligned}
$$

If the minimizing sequence $\left\{v_{n}\right\}$ of $\hat{e}_{\lambda}(\alpha)$ satisfies

$$
\liminf _{n \rightarrow \infty} \int_{\mathbb{R}^{d}} \int_{\mathbb{R}^{d}} \frac{v_{n}^{2}(x) v_{n}^{2}(y)}{|x-y|^{2}} d x d y \geq C_{0}>0
$$

then the claim (2.14) follows in view of (2.15). In order to prove (2.16), since $\hat{e}_{\lambda}(N)<0$, we obtain from the Gagliardo-Nirenberg inequality (1.13) that

$$
\begin{aligned}
& \lambda \liminf _{n \rightarrow \infty} \int_{\mathbb{R}^{d}}[1-g(x)] v_{n}^{2}(x) d x \\
= & \liminf _{n \rightarrow \infty}\left\{\int_{\mathbb{R}^{d}}\left|\nabla v_{n}(x)\right|^{2} d x-\frac{1}{2} \int_{\mathbb{R}^{d}} \int_{\mathbb{R}^{d}} \frac{v_{n}^{2}(x) v_{n}^{2}(y)}{|x-y|^{2}} d x d y\right\}-\hat{e}_{\lambda}(N) \\
\geq & \left(1-\frac{N}{N^{*}}\right) \liminf _{n \rightarrow \infty} \int_{\mathbb{R}^{d}}\left|\nabla v_{n}(x)\right|^{2} d x-\hat{e}_{\lambda}(N)>0 .
\end{aligned}
$$

Following the above inequality, since $1-g(x) \in L^{\frac{d}{2}}\left(\mathbb{R}^{d}\right)$, we deduce that there exist constants $\varepsilon>0$ and large $R>0$ such that

$$
\liminf _{n \rightarrow \infty} \int_{B_{R}(0)} v_{n}^{2}(x) \geq \varepsilon
$$

from which the estimate (2.16) follows and the claim (2.14) is hence established.

Once (2.14) holds, we can follow Ref. 19, Lemma II.1 to obtain that the following sub-additivity condition holds:

$$
\hat{e}_{\lambda}(N)<\hat{e}_{\lambda}(\alpha)+\hat{e}_{\lambda}(N-\alpha) .
$$


By contradiction, we now suppose that the dichotomy occurs. It then follows from the arguments of Ref. 19, Lemma III.1 that for $k>0$ large enough, there exist two sequences $\left\{u_{n_{k, 1}}\right\}$ and $\left\{u_{n_{k, 2}}\right\}$ such that $u_{n_{k}}$ satisfies

$$
\begin{gathered}
\left\|u_{n_{k}}-\left(u_{n_{k, 1}}+u_{n_{k, 2}}\right)\right\|_{q} \rightarrow 0 \text { as } k \rightarrow \infty \text { for } 2 \leq q<2^{*}, \\
\left|\int_{\mathbb{R}^{d}} u_{n_{k, 1}}^{2}-\alpha\right| \rightarrow 0 \text { and }\left|\int_{\mathbb{R}^{d}} u_{n_{k, 2}}^{2}-(N-\alpha)\right| \rightarrow 0 \text { as } k \rightarrow \infty, \\
\operatorname{dist}\left(\operatorname{Supp} u_{n_{k, 1}}, \operatorname{Supp} u_{n_{k, 2}}\right) \rightarrow \infty \text { as } k \rightarrow \infty, \\
\liminf _{k \rightarrow \infty} \int_{\mathbb{R}^{d}}\left\{\left|\nabla u_{n_{k}}\right|^{2}-\left|\nabla u_{n_{k, 1}}\right|^{2}-\left|\nabla u_{n_{k, 2}}\right|^{2}\right\} d x \geq 0 .
\end{gathered}
$$

These further imply that

$$
\begin{aligned}
& \int_{\mathbb{R}^{d}} \int_{\mathbb{R}^{d}} \frac{u_{n_{k}}^{2}(x) u_{n_{k}}^{2}(y)}{|x-y|^{2}} d x d y \\
= & \int_{\mathbb{R}^{d}} \int_{\mathbb{R}^{d}} \frac{u_{n_{k, 1}}^{2}(x) u_{n_{k, 1}}^{2}(y)}{|x-y|^{2}} d x d y+\int_{\mathbb{R}^{d}} \int_{\mathbb{R}^{d}} \frac{u_{n_{k, 2}}^{2}(x) u_{n_{k, 2}}^{2}(y)}{|x-y|^{2}} d x d y+o(1)
\end{aligned}
$$

as $k \rightarrow \infty$, and therefore,

$$
\begin{aligned}
\hat{e}_{\lambda}(N)= & \lim _{k \rightarrow \infty} \hat{E}_{\lambda}\left(u_{n_{k}}\right) \\
\geq & \lim _{k \rightarrow \infty}\left[\int_{\mathbb{R}^{d}}\left(\left|\nabla u_{n_{k, 1}}\right|^{2}+\left|\nabla u_{n_{k, 1}}\right|^{2}\right) d x-\lambda \int_{\mathbb{R}^{d}}[1-g(x)]\left(u_{n_{k, 1}}^{2}+u_{n_{k, 2}}^{2}\right) d x\right. \\
& \left.-\frac{1}{2} \int_{\mathbb{R}^{d}} \int_{\mathbb{R}^{d}} \frac{u_{n_{k, 1}}^{2}(x) u_{n_{k, 1}}^{2}(y)}{|x-y|^{2}} d x d y-\frac{1}{2} \int_{\mathbb{R}^{d}} \int_{\mathbb{R}^{d}} \frac{u_{n_{k, 2}}^{2}(x) u_{n_{k, 2}}^{2}(y)}{|x-y|^{2}} d x d y\right]+o(1) \\
\geq & \hat{e}_{\lambda}(\alpha)+\hat{e}_{\lambda}(N-\alpha),
\end{aligned}
$$

which however contradicts (2.18). Therefore, the dichotomy cannot occur, either.

By the concentration-compactness lemma (cf. Ref. 19), we now conclude that only the compactness occurs for the subsequence $\left\{u_{n_{k}}\right\}$. This implies that there exists a subsequence still denoted by $\left\{u_{n_{k}}\right\}$ and $\left\{y_{k}\right\}$ such that the sequence $\hat{u}_{k}(\cdot):=u_{n_{k}}\left(\cdot+y_{k}\right)$ satisfies

$$
\hat{u}_{n_{k}} \rightarrow u_{0} \text { weakly in } H^{1}\left(\mathbb{R}^{d}\right)
$$

and

$$
\hat{u}_{k} \rightarrow u_{0} \text { strongly in } L^{q}\left(\mathbb{R}^{d}\right) \text { for } 2 \leq q<2^{*}
$$

for some $u_{0} \in H^{1}\left(\mathbb{R}^{d}\right)$. So, we have

$$
\lim _{k \rightarrow \infty} \int_{\mathbb{R}^{d}} \int_{\mathbb{R}^{d}} \frac{\hat{u}_{k}^{2}(x) \hat{u}_{k}^{2}(y)}{|x-y|^{2}} d x d y=\int_{\mathbb{R}^{d}} \int_{\mathbb{R}^{d}} \frac{u_{0}^{2}(x) u_{0}^{2}(y)}{|x-y|^{2}} d x d y .
$$

By the weak lower semi-continuity, we also get that $\liminf _{k \rightarrow \infty}\left\|\nabla \hat{u}_{k}\right\|_{2}^{2} \geq\left\|\nabla u_{0}\right\|_{2}^{2}$, and so $u_{0}$ must be a minimizer of $\hat{e}_{\lambda}$, which further implies that $\lim _{k \rightarrow \infty}\left\|\nabla \hat{u}_{k}\right\|_{2}^{2}=\left\|\nabla u_{0}\right\|_{2}^{2}$. Next, we claim that the translation $\left\{y_{k}\right\}$ is bounded uniformly for $k$. Actually, if the claim is false, we then have

$$
\begin{aligned}
\hat{e}_{\lambda}(N) & =\lim _{k \rightarrow \infty} \hat{E}_{\lambda}\left(u_{n_{k}}\right) \\
& =\lim _{k \rightarrow \infty}\left(\int_{\mathbb{R}^{d}}\left|\nabla u_{n_{k}}\right|^{2} d x-\lambda \int_{\mathbb{R}^{d}}[1-g(x)] u_{n_{k}}^{2} d x-\frac{1}{2} \int_{\mathbb{R}^{d}} \frac{u_{n_{k}}^{2}(x) u_{n_{k}}^{2}(y)}{|x-y|^{2}} d x d y\right) \\
& =\lim _{k \rightarrow \infty}\left(\int_{\mathbb{R}^{d}}\left|\nabla \hat{u}_{n_{k}}\right|^{2} d x-\lambda \int_{\mathbb{R}^{d}}\left[1-g\left(x+y_{k}\right)\right] \hat{u}_{n_{k}}^{2} d x-\frac{1}{2} \int_{\mathbb{R}^{d}} \frac{\hat{u}_{k}^{2}(x) \hat{u}_{k}^{2}(y)}{|x-y|^{2}} d x d y\right) \\
& =\int_{\mathbb{R}^{d}}\left|\nabla u_{0}\right|^{2} d x-\frac{1}{2} \int_{\mathbb{R}^{d}} \frac{u_{0}^{2}(x) u_{0}^{2}(y)}{|x-y|^{2}} d x d y>\hat{E}_{\lambda}\left(u_{0}\right)=\hat{e}_{\lambda}(N),
\end{aligned}
$$

which is a contradiction. Once $\left\{y_{k}\right\}$ is bounded uniformly for $k$, taking a subsequence if necessary, we may assume that $\lim _{k \rightarrow \infty} y_{k}=y_{0}$, from which we obtain that

$$
u_{n_{k}} \rightarrow u_{0}\left(\cdot-y_{0}\right) \text { strongly in } L^{q}\left(\mathbb{R}^{d}\right) \text { as } k \rightarrow \infty \text {, where } 2 \leq q<2^{*} .
$$


This implies that $u_{0}\left(\cdot-y_{0}\right)$ is therefore a minimizer of $\hat{e}_{\lambda}(N)$, and we are done.

We next define $\lambda^{*}(N)$ by

$$
\lambda^{*}(N)=\inf _{\left\{u \in H^{1}\left(\mathbb{R}^{d}\right),\|u\|_{2}^{2}=N\right\}} F(u), 0<N<N^{*},
$$

where $F(u)$ satisfies

$$
F(u)=\frac{\int_{\mathbb{R}^{d}}|\nabla u|^{2} d x-\frac{1}{2} \int_{\mathbb{R}^{d}} \int_{\mathbb{R}^{d}} \frac{u^{2}(x) u^{2}(y)}{|x-y|^{2}} d x d y}{\int_{\mathbb{R}^{d}}(1-g) u^{2} d x} .
$$

The following lemma gives the existence and estimates of $\lambda^{*}(N)$.

Lemma 2.3. For any $0<N<N^{*}$, let $\lambda^{*}(N)$ be defined by (2.19), where $g(x)$ satisfies $\left(M_{1}\right)$. Then $\lambda^{*}(N)$ satisfies

$$
0<\frac{S_{d}\left(N^{*}-N\right)}{N^{*}\|1-g\|_{\frac{d}{2}}} \leq \lambda^{*}(N) \leq \frac{N^{*}-N}{\int_{\mathbb{R}^{d}}(1-g) Q^{2} d x}<\infty,
$$

where $S_{d}>0$ is the optimal constant of the Sobolev inequality (1.14).

Proof. By the definition of $\lambda^{*}(N)$, we deduce from (1.11) that

$$
\lambda^{*}(N) \leq F\left(\sqrt{\frac{N}{N^{*}}} Q\right)=\frac{\left(N^{*}-N\right) \int_{\mathbb{R}^{d}}|\nabla Q|^{2} d x}{N^{*} \int_{\mathbb{R}^{d}}(1-g) Q^{2} d x}=\frac{N^{*}-N}{\int_{\mathbb{R}^{d}}(1-g) Q^{2} d x},
$$

which implies the upper estimate of (2.21). As for the lower estimate of (2.21), by the GagliardoNirenberg inequality (1.13), we derive that for any $u \in H^{1}\left(\mathbb{R}^{d}\right)$,

$$
F(u) \geq \frac{\left(1-\frac{N}{N^{*}}\right) \int_{\mathbb{R}^{d}}|\nabla u|^{2} d x}{\int_{\mathbb{R}^{d}}(1-g) u^{2} d x} \geq \frac{\left(1-\frac{N}{N^{*}}\right) \int_{\mathbb{R}^{d}}|\nabla u|^{2} d x}{\|1-g\|_{\frac{d}{2}}\|u\|_{2^{*}}^{2}} \geq \frac{S_{d}\left(N^{*}-N\right)}{N^{*}\|1-g\|_{\frac{d}{2}}},
$$

where the Sobolev inequality (1.14) is used in the last inequality. We thus obtain from above that the lower estimate of (2.21) holds, and the lemma is therefore proved.

Proof of Theorem 1.1. By Lemma 2.1(i), in the following, we only need to prove Theorem 1.1(ii).

First, we consider the case where $0<N<N^{*}$ and $\lambda>\lambda^{*}(N)$. By setting $\eta=\frac{\lambda-\lambda^{*}(N)}{2}>0$, there exists $u_{0} \in H^{1}\left(\mathbb{R}^{d}\right)$ satisfying $\left\|u_{0}\right\|_{2}^{2}=N$ such that

$$
\lambda^{*}(N) \leq F\left(u_{0}\right)<\lambda^{*}(N)+\frac{\eta}{2},
$$

from which we infer that

$$
\begin{aligned}
\hat{e}_{\lambda}(N) & \leq \hat{E}_{\lambda}\left(u_{0}\right)<\left(\lambda^{*}(N)+\frac{\eta}{2}-\lambda\right) \int_{\mathbb{R}^{d}}(1-g) u_{0}^{2} \\
& =-\frac{3 \eta}{2} \int_{\mathbb{R}^{d}}(1-g) u_{0}^{2}<0 .
\end{aligned}
$$

Applying Lemma 2.2, we then conclude from (2.22) that there exists a minimizer for (2.1) in this case, which therefore gives the existence of Theorem 1.1(ii).

Next, we consider the case where $0<N<N^{*}$ and $0<\lambda<\lambda^{*}(N)$. By the definition of $\lambda^{*}(N)$, in this case, we note that

$$
\begin{aligned}
\hat{E}_{\lambda}(u) & \geq \lambda^{*}(N) \int_{\mathbb{R}^{d}}(1-g) u^{2} d x-\lambda \int_{\mathbb{R}^{d}}(1-g) u^{2} d x \\
& =\left(\lambda^{*}(N)-\lambda\right) \int_{\mathbb{R}^{d}}(1-g) u^{2} d x \geq 0,
\end{aligned}
$$

which implies that $\hat{e}_{\lambda}(N) \geq 0$. Since Lemma 2.1(ii) gives that $\hat{e}_{\lambda}(N) \leq 0$, we have $\hat{e}_{\lambda}(N)=0$. If (2.1) has a minimizer $u_{0}$, we then have

$$
0=\hat{e}_{\lambda}(N)=\hat{E}_{\lambda}\left(u_{0}\right) \geq\left(\lambda^{*}(N)-\lambda\right) \int_{\mathbb{R}^{d}}(1-g) u_{0}^{2} d x \geq 0,
$$


which implies that $\int_{\mathbb{R}^{d}}(1-g) u_{0}^{2} d x=0$. However, this is impossible since $1-g \geq 0$ and $g(x) \neq 1$ in $\mathbb{R}^{d}$ and $\left\|u_{0}\right\|_{2}^{2}=N$. Therefore, we have the nonexistence of minimizers for the case where $0<N<N^{*}$ and $0<\lambda<\lambda^{*}(N)$. This establishes the nonexistence of Theorem 1.1(ii), and we are done.

\section{LIMIT BEHAVIOR OF MINIMIZERS AS $\lambda \rightarrow \infty$}

This section is devoted to the proof of Theorem 1.2 on the limit behavior of positive minimizers for $e_{\lambda}(N)$ as $\lambda \rightarrow \infty$, for the case where $0<N<N^{*}$, and $g(x) \in C_{l o c}^{\alpha}\left(\mathbb{R}^{d}\right)$ satisfies $\left(\mathrm{M}_{1}\right)$ and $\left(\mathrm{M}_{2}\right)$ for $p>0$. In this case, we recall from Theorem 1.1 that there exists at least one minimizer $u_{\lambda}$ for $e_{\lambda}(N)$, i.e.,

$$
e_{\lambda}(N)=\inf _{\left\{u \in H^{1}\left(\mathbb{R}^{d}\right),\|u\|_{2}^{2}=N\right\}} E_{\lambda}(u)=E_{\lambda}\left(u_{\lambda}\right) .
$$

We also introduce the following related minimization problem, which can be thought of as the "limit" case of $e_{\lambda}(N)$ for $\lambda \rightarrow \infty$ :

$$
e_{\infty}(N)=\inf _{\left\{u \in \mathcal{H},\|u\|_{2}^{2}=N\right\}} E_{\infty}(u),
$$

where the space $\mathcal{H}$ is defined by

$$
\mathcal{H}=\left\{u \in H^{1}\left(\mathbb{R}^{d}\right): \int_{\mathbb{R}^{d}}|x|^{p} u^{2} d x<\infty\right\}, p>0
$$

and the energy functional $E_{\infty}(u)$ is of the form

$$
E_{\infty}(u)=\int_{\mathbb{R}^{d}}|\nabla u|^{2} d x+\int_{\mathbb{R}^{d}}|x|^{p} u^{2} d x-\frac{1}{2} \int_{\mathbb{R}^{d}} \int_{\mathbb{R}^{d}} \frac{u^{2}(x) u^{2}(y)}{|x-y|^{2}} d x d y, \quad u \in \mathcal{H} .
$$

Similar to Refs. 10 and 12, one can prove that $e_{\infty}(N)$ admits positive minimizers if and only if $N$ $\in\left(0, N^{*}\right)$, where $N^{*}=\|Q\|_{2}^{2}$ and $Q>0$ is a ground state of (1.5). We leave the detailed proof to the interested reader. Moreover, we have the following qualitative properties of minimizers for $e_{\infty}(N)$, which play an important role in analyzing the limiting behavior of minimizers $u_{\lambda}$ for $e_{\lambda}(N)$.

Proposition 3.1. For any $N \in\left(0, N^{*}\right)$, the following properties hold true:

1. $\frac{e_{\infty}(N)}{N} \leq C(p)$, where $C(p)$ depends only on $p$.

2. Any positive minimizer $U_{N}$ of $e_{\infty}(N)$ must be radially symmetric and strictly decreasing in $|x|$. Moreover, $U_{N}$ satisfies the following exponential decay:

$$
U_{N}(x) \leq C e^{-|x|} \text { as }|x| \rightarrow \infty,
$$

where $C>0$ is independent of $x$.

Proof. 1. By a transform $v=u N^{-\frac{1}{2}}$, for any $0<N<N^{*}$, the minimization problem $e_{\infty}(N)$ is reduced equivalently to the following one:

$$
e_{N}=\inf _{\left\{v \in \mathcal{H},\|v\|_{2}^{2}=1\right\}} \mathcal{E}_{N}(v)
$$

where the energy functional $\mathcal{E}_{N}(v)$ is defined by

$$
\mathcal{E}_{N}(v)=\int_{\mathbb{R}^{d}}|\nabla v|^{2} d x+\int_{\mathbb{R}^{d}}|x|^{p} v^{2} d x-\frac{N}{2} \int_{\mathbb{R}^{d}} \int_{\mathbb{R}^{d}} \frac{v^{2}(x) v^{2}(y)}{|x-y|^{2}} d x d y, \quad p>0 .
$$

It is clear that $e_{N}=\frac{e_{\infty}(N)}{N}$. By the arguments of Refs. 10 and 12, one can deduce that there exists a constant $C(p)>0$ depending only on $p$ such that $e_{N} \leq C(p)$ and hence $\frac{e_{\infty}(N)}{N} \leq C(p)$ for any $0<N<N^{*}$.

2. Using the symmetric-decreasing rearrangement, similar to Refs. 5 and 16, one can obtain that any positive minimizer $U_{N}(x)$ of $e_{\infty}(N)$ must be radially symmetric and decreasing in $|x|$. Furthermore, since $\frac{e_{\infty}(N)}{N} \leq C(p)$ for any $0<N<N^{*}$, similar to the proof of (3.35), one can follow the comparison principle to obtain that $U_{N}(x)$ decays exponentially as $|x| \rightarrow \infty .6,9,11,18,22$ 
We finally prove that $U_{N}(x)$ strictly decreases in $|x|$. On the contrary, suppose that there exist two points $y_{1} \in \mathbb{R}^{d}$ and $y_{2} \in \mathbb{R}^{d}$ such that $\left|y_{1}\right| \neq\left|y_{2}\right|$ and $U_{N}\left(y_{1}\right)=U_{N}\left(y_{2}\right)$. Without loss of generality, we may assume $0 \leq a=\left|y_{1}\right|<b=\left|y_{2}\right|$. Since $U_{N}(x)>0$ decreases in $|x|$, we have $U_{N}(x) \equiv$ const. $>0$ in the annular domain $\bar{\Omega}:=\bar{B}_{b}(0) / B_{a}(0)$. Hence, we derive from $(1.18)$ that $-\Delta U_{N}(x) \equiv 0$ in $\bar{\Omega}$ and

$$
|x|^{p} U_{N}(x)=\mu U_{N}(x)+\left(\int_{\mathbb{R}^{d}} \frac{U_{N}^{2}(y)}{|x-y|^{2}} d y\right) U_{N}(x) \text { in } \bar{\Omega} .
$$

Applying (3.6), we have

$$
f(x)=\int_{\mathbb{R}^{d}} \frac{U_{N}^{2}(y)}{|x-y|^{2}} d y-|x|^{p}+\mu \equiv 0 \quad \text { in } \bar{\Omega},
$$

which thus gives that

$$
f\left(P_{1}\right)=f\left(P_{2}\right), \quad \text { where } P_{1}=(a, 0, \ldots, 0), P_{2}=(b, 0, \ldots, 0) .
$$

On the other hand, for any point $y=\left(y_{1}, y_{2}, \ldots, y_{d}\right) \in \mathbb{R}^{d}$, set $P=\left(\frac{a+b}{2}, 0, \ldots, 0\right)$ and define $y_{P}$ $=\left(a+b-y_{1}, y_{2}, \ldots, y_{d}\right)$ such that

$$
\left|P_{1}-y\right|^{2} \leq\left|P_{2}-y\right|^{2} \text { and }|y|<\left|y_{P}\right| \text { in }\left\{y \in \mathbb{R}^{d}: y_{1} \leq \frac{a+b}{2}\right\} .
$$

By direct calculations, we then deduce that

$$
\begin{aligned}
& f\left(P_{1}\right)-f\left(P_{2}\right) \\
= & \int_{\mathbb{R}^{d}}\left[\frac{U_{N}^{2}(y)}{\left|P_{1}-y\right|^{2}}-\frac{U_{N}^{2}(y)}{\left|P_{2}-y\right|^{2}}\right] d y+\left(b^{q}-a^{q}\right) \\
= & \int_{\left\{y \in \mathbb{R}^{d}: y_{1} \leq \frac{a+b}{2}\right\}} U_{N}^{2}(y)\left[\frac{1}{\left|P_{1}-y\right|^{2}}-\frac{1}{\left|P_{2}-y\right|^{2}}\right] d y \\
& +\int_{\left\{y \in \mathbb{R}^{d}: y_{1}>\frac{a+b}{2}\right\}} U_{N}^{2}(y)\left[\frac{1}{\left|P_{1}-y\right|^{2}}-\frac{1}{\left|P_{2}-y\right|^{2}}\right] d y+\left(b^{q}-a^{q}\right) \\
= & \int_{\left\{y \in \mathbb{R}^{d}: y_{1} \leq \frac{a+b}{2}\right\}} U_{N}^{2}(y)\left[\frac{1}{\left|P_{1}-y\right|^{2}}-\frac{1}{\left|P_{2}-y\right|^{2}}\right] d y \\
& +\int_{\left\{y \in \mathbb{R}^{d}: y_{1}<\frac{a+b}{2}\right\}} U_{N}^{2}\left(y_{P}\right)\left[\frac{1}{\left|P_{2}-y\right|^{2}}-\frac{1}{\left|P_{1}-y\right|^{2}}\right] d y+\left(b^{q}-a^{q}\right) \\
= & \int_{\left\{y \in \mathbb{R}^{d}: y_{1} \leq \frac{a+b}{2}\right\}}\left[U_{N}^{2}(y)-U_{N}^{2}\left(y_{P}\right)\right]\left[\frac{1}{\left|P_{1}-y\right|^{2}}-\frac{1}{\left|P_{2}-y\right|^{2}}\right] d y+\left(b^{q}-a^{q}\right)>0,
\end{aligned}
$$

since $U_{N}(x)$ decreases to zero in $|x|$. We thus conclude from (3.9) that $f\left(P_{1}\right)>f\left(P_{2}\right)$, which however contradicts (3.8). Therefore, $U_{N}(x)$ strictly decreases in $|x|$, which completes the proof.

We next apply the above qualitative properties of $e_{\infty}(N)$ to address the following refined energy estimates.

Lemma 3.2. Suppose $g(x)$ satisfies $\left(\mathrm{M}_{1}\right)$ and $\left(\mathrm{M}_{2}\right)$ for $p>0$. Then for any $N \in\left(0, N^{*}\right)$, we have

$$
B(p)\left(1-\frac{N}{N^{*}}\right)^{\frac{p}{2+p}} N \lambda^{\frac{2}{2+p}} \leq e_{\lambda}(N) \leq \lambda^{\frac{2}{2+p}}\left[e_{\infty}(N)+o(1)\right] \text { as } \lambda \rightarrow \infty,
$$

where the constant $B(p)>0$ depends only on $p$.

Proof. By Proposition 3.1, let $U_{N}>0$ be a positive minimizer of $e_{\infty}(N)$ for any $N \in\left(0, N^{*}\right)$, where the problem $e_{\infty}(N)$ is defined in (3.1). Take the trial function $\hat{U}_{N}(x)=\lambda^{\frac{d}{2(2+p)}} U_{N}\left(\lambda^{\frac{1}{2+p}} x\right)$. We 
then have

$$
\begin{aligned}
& e_{\lambda}(N) \leq E_{\lambda}\left(\hat{U}_{N}\right) \\
= & \lambda^{\frac{2}{2+p}}\left[\int_{\mathbb{R}^{d}}\left|\nabla U_{N}(x)\right|^{2} d x+\lambda^{\frac{p}{2+p}} \int_{\mathbb{R}^{d}} g\left(\frac{x}{\lambda^{\frac{1}{2+p}}}\right) U_{N}^{2}(x) d x\right. \\
& \left.\quad-\frac{1}{2} \int_{\mathbb{R}^{d}} \int_{\mathbb{R}^{d}} \frac{U_{N}^{2}(x) U_{N}^{2}(y)}{|x-y|^{2}} d x d y\right] \\
= & \lambda^{\frac{2}{2+p}}\left(e_{\infty}(N)+\int_{\mathbb{R}^{d}}\left[\lambda^{\frac{p}{2+p}} g\left(\frac{x}{\lambda^{\frac{1}{2+p}}}\right)-|x|^{p}\right] U_{N}^{2}(x) d x\right) \text { as } \lambda \rightarrow \infty .
\end{aligned}
$$

Since $g(x)$ satisfies $\left(\mathrm{M}_{1}\right)$ and $\left(\mathrm{M}_{2}\right)$ for $p>0$, we deduce that there exists a large constant $C>0$ such that

$$
g(x) \leq C|x|^{p} \text { and } \lambda^{\frac{p}{2+p}} g\left(\frac{x}{\lambda^{\frac{1}{2+p}}}\right) \leq C|x|^{p} \text { in } \mathbb{R}^{d} .
$$

Since Proposition 3.1 gives that $U_{N}(x)$ decays exponentially as $|x| \rightarrow \infty$, we have

$$
\int_{|x|>\lambda^{\frac{1}{2(2+p)}}}\left[\lambda^{\frac{p}{2+p}} g\left(\frac{x}{\lambda^{\frac{1}{2+p}}}\right)-|x|^{p}\right] U_{N}^{2}(x) d x=o(1) \text { as } \lambda \rightarrow \infty .
$$

We thus calculate that

$$
\begin{aligned}
& \int_{\mathbb{R}^{d}}\left[\lambda^{\frac{p}{2+p}} g\left(\frac{x}{\lambda^{\frac{1}{2+p}}}\right)-|x|^{p}\right] U_{N}^{2}(x) d x \\
= & \int_{|x| \leq \lambda^{\frac{1}{2(2+p)}}}\left[\lambda^{\frac{p}{2+p}} g\left(\frac{x}{\lambda^{\frac{1}{2+p}}}\right)-|x|^{p}\right] U_{N}^{2}(x) d x \\
& +\int_{|x|>\lambda^{\frac{1}{2(2+p)}}}\left[\lambda^{\frac{p}{2+p}} g\left(\frac{x}{\lambda^{\frac{1}{2+p}}}\right)-|x|^{p}\right] U_{N}^{2}(x) d x \\
= & (1+o(1)-1) \int_{|x| \leq \lambda^{\frac{1}{2(2+p)}}|x|^{p}} U_{N}^{2}(x) d x+o(1)=o(1) \text { as } \lambda \rightarrow \infty,
\end{aligned}
$$

from which we obtain the upper energy estimate of (3.10).

Next, we shall address the lower estimate of (3.10). By the Gagliardo-Nirenberg inequality (1.13), we deduce from the upper energy estimate of (3.10) that

$$
\lambda \int_{\mathbb{R}^{d}} g(x) u_{\lambda}^{2} d x \leq e_{\lambda}(N) \leq\left[e_{\infty}(N)+o(1)\right] \lambda^{\frac{2}{2+p}} \text { as } \lambda \rightarrow \infty,
$$

which implies that

$$
\int_{\mathbb{R}^{d}} g(x) u_{\lambda}^{2} d x \rightarrow 0 \text { as } \lambda \rightarrow \infty .
$$

On the other hand, under the assumption $\left(\mathrm{M}_{2}\right)$ for $p>0$, let $\varepsilon_{0}>0$ be small enough that $g(x) \geq \frac{1}{2}|x|^{p}$ holds for any $|x| \leq 2 \varepsilon_{0}$. Because 0 is the unique global minimum point of $g(x)$ satisfying $g(0)=0$, we obtain from (3.13) that

$$
\int_{\left\{|x|>\varepsilon_{0}\right\}} u_{\lambda}^{2} d x \rightarrow 0 \text { as } \lambda \rightarrow \infty
$$

and

$$
\int_{\left\{|x| \leq \varepsilon_{0}\right\}} u_{\lambda}^{2} d x=\int_{\mathbb{R}^{d}} u_{\lambda}^{2}-\int_{\left\{|x|>\varepsilon_{0}\right\}} u_{\lambda}^{2} d x \geq \frac{N}{4} \text { as } \lambda \rightarrow \infty .
$$

Define now $\hat{u}_{\lambda}=\varphi u_{\lambda}$, where $0 \leq \varphi \in C_{0}^{\infty}\left(\mathbb{R}^{d}\right)$ is a smooth cut-off function satisfying

$$
\left\{\begin{array}{l}
\varphi=1 \text { for }|x| \leq \varepsilon_{0} \\
0<\varphi<1 \text { for } \varepsilon_{0}<|x|<2 \varepsilon_{0} \\
\varphi=0 \text { for }|x| \geq 2 \varepsilon_{0} ;|\nabla \varphi| \leq \frac{M}{\varepsilon_{0}} \text { in } \mathbb{R}^{d} .
\end{array} .\right.
$$


One can check that $\hat{u}_{\lambda}$ satisfies

$$
\left\{\begin{array}{l}
0 \leq \hat{u}_{\lambda}(x) \leq u_{\lambda}(x) \text { in } \mathbb{R}^{d} \\
\frac{N}{4} \leq\left\|\hat{u}_{\lambda}\right\|_{2}^{2} \leq\left\|u_{\lambda}\right\|_{2}^{2} \\
\left\|\nabla \hat{u}_{\lambda}\right\|_{2}^{2} \leq 2\left\|\nabla u_{\lambda}\right\|_{2}^{2}+\frac{2 M^{2}}{\varepsilon_{0}^{2}} N .
\end{array}\right.
$$

By the Caffarelli-Kohn-Nirenberg inequality (cf. Ref. 3), we then deduce from (3.15) that there exists a constant $A(p)>0$ depending only on $p$ such that

$$
\begin{aligned}
\left\|\nabla \hat{u}_{\lambda}\right\|_{2}^{\frac{p}{2+p}}\left\|g^{\frac{1}{2}}(x) \hat{u}_{\lambda}\right\|_{2}^{\frac{2}{2+p}} & \geq\left(\frac{1}{2}\right)^{\frac{1}{2+p}}\left\|\nabla \hat{u}_{\lambda}\right\|_{2}^{\frac{p}{2+p}}\left\||x|^{\frac{p}{2}} \hat{u}_{\lambda}\right\|_{2}^{\frac{2}{2+p}} \\
& \geq\left(\frac{1}{2}\right)^{\frac{1}{2+p}} A(p)\left\|\hat{u}_{\lambda}\right\|_{2} \geq\left(\frac{1}{2}\right)^{1+\frac{1}{2+p}} A(p) N^{\frac{1}{2}} .
\end{aligned}
$$

Since it yields from (3.13) that

$$
\int_{\mathbb{R}^{d}} g(x) \hat{u}_{\lambda}^{2}(x) \leq \int_{\mathbb{R}^{d}} g(x) u_{\lambda}^{2}(x) \rightarrow 0 \text { as } \lambda \rightarrow \infty,
$$

we obtain from (3.16) that

$$
\left\|\nabla \hat{u}_{\lambda}\right\|_{2} \rightarrow \infty \text { as } \lambda \rightarrow \infty .
$$

By (3.15) and (3.17), we then have

$$
\left\|\nabla u_{\lambda}\right\|_{2}^{2} \geq \frac{1}{2}\left\|\nabla \hat{u}_{\lambda}\right\|_{2}^{2}-\frac{M^{2} N}{\varepsilon_{0}^{2}} \geq \frac{1}{4}\left\|\nabla \hat{u}_{\lambda}\right\|_{2}^{2} \text { as } \lambda \rightarrow \infty .
$$

Following (3.15), (3.16), and (3.18), we thus conclude that

$$
\begin{aligned}
\left\|\nabla u_{\lambda}\right\|_{2}^{\frac{p}{2+p}}\left\|g^{\frac{1}{2}}(x) u_{\lambda}\right\|_{2}^{\frac{2}{2+p}} & \geq\left(\frac{1}{4}\left\|\nabla \hat{u}_{\lambda}\right\|_{2}^{2}\right)^{\frac{p}{2(2+p)}}\left\|g^{\frac{1}{2}}(x) \hat{u}_{\lambda}\right\|_{2}^{\frac{2}{2+p}} \\
& \geq\left(\frac{1}{2}\right)^{1+\frac{p+1}{2+p}} A(p) N^{\frac{1}{2}} \text { as } \lambda \rightarrow \infty .
\end{aligned}
$$

By the Gagliardo-Nirenberg inequality (1.13), together with Young's inequality, we now deduce from (3.19) that

$$
\begin{aligned}
e_{\lambda}(N) & \geq\left(1-\frac{N}{N^{*}}\right)\left\|\nabla u_{\lambda}\right\|_{2}^{2}+\lambda\left\|g^{\frac{1}{2}}(x) u_{\lambda}\right\|_{2}^{2} \\
& \geq(2+p)\left(4 p^{p}\right)^{-\frac{1}{2+p}}\left(1-\frac{N}{N^{*}}\right)^{\frac{p}{2+p}} \lambda^{\frac{2}{2+p}}\left\|\nabla u_{\lambda}\right\|_{2}^{\frac{2 p}{2+p}}\left\|g^{\frac{1}{2}}(x) u_{\lambda}\right\|_{2}^{\frac{4}{2+p}} \\
& \geq \frac{1}{16}(2+p) p^{-\frac{p}{2+p}} A^{2}(p) N\left(1-\frac{N}{N^{*}}\right)^{\frac{p}{2+p}} \lambda^{\frac{2}{2+p}} \\
& :=B(p)\left(1-\frac{N}{N^{*}}\right)^{\frac{p}{2+p}} N \lambda^{\frac{2}{2+p}} \text { as } \lambda \rightarrow \infty,
\end{aligned}
$$

which then implies that the lower bound estimate of (3.10) also holds.

Using (3.10) and (3.19), one can establish immediately the following corollary.

Corollary 3.3. Suppose $g(x)$ satisfies $\left(\mathrm{M}_{1}\right)$ and $\left(\mathrm{M}_{2}\right)$ for $p>0$ and let $u_{\lambda}>0$ be a positive minimizer of $e_{\lambda}(N)$. Then for any $N \in\left(0, N^{*}\right)$, we have

$$
\left\{\begin{array}{l}
m_{1}(N, p) \lambda^{\frac{2}{2+p}} \leq\left\|\nabla u_{\lambda}\right\|_{2}^{2} \leq m_{2}(N, p) \lambda^{\frac{2}{2+p}} \text { as } \lambda \rightarrow \infty, \\
M_{1}(N, p) \lambda^{\frac{2}{2+p}} \leq \lambda\left\|g^{\frac{1}{2}}(x) u_{\lambda}\right\|_{2}^{2} \leq M_{2}(N, p) \lambda^{\frac{2}{2+p}} \text { as } \lambda \rightarrow \infty,
\end{array},\right.
$$

where $m_{1}(N, p), m_{2}(N, p), M_{1}(N, p)$, and $M_{2}(N, p)$ are positive constants depending only on $N$ and $p$. 
Proof. Indeed, the upper bound estimates of (3.21) follow directly from Lemma 3.2 and the Gagliardo-Nirenberg inequality (1.13) as well. As for the lower bound estimates of (3.21), by contradiction, we first suppose that $m_{1}(N, p)=0$, i.e., suppose there exists a sequence $\left\{\lambda_{n}\right\}$, where $\lambda_{n}$ $\rightarrow \infty$ as $n \rightarrow \infty$, such that

$$
\lim _{\lambda_{n} \rightarrow \infty} \frac{\left\|\nabla u_{\lambda_{n}}\right\|_{2}^{2}}{\lambda_{n}^{\frac{2}{2+p}}}=0 .
$$

Following (3.19) and (3.22), we then obtain that

$$
\lim _{\lambda_{n} \rightarrow \infty} \frac{\lambda_{n}\left\|g^{\frac{1}{2}}(x) u_{\lambda_{n}}\right\|_{2}^{2}}{\lambda_{n}^{\frac{2}{2+p}}}=\lim _{\lambda_{n} \rightarrow \infty}\left\|g^{\frac{1}{2}}(x) u_{\lambda_{n}}\right\|_{2}^{2} \lambda_{n}^{\frac{p}{2+p}}=\infty,
$$

which however contradicts (3.10). Therefore, we have $m_{1}>0$. Similarly, one can also obtain that $M_{1}$ $>0$ and therefore (3.21) is proved.

Since $u_{\lambda}>0$ is a positive minimizer of $E_{\lambda}(u)$, it satisfies the following Euler-Lagrange equation:

$$
-\Delta u_{\lambda}+\lambda g(x) u_{\lambda}=\mu_{\lambda} u_{\lambda}+\left(\int_{\mathbb{R}^{d}} \frac{u_{\lambda}^{2}(y)}{|x-y|^{2}} d y\right) u_{\lambda} \text { in } \mathbb{R}^{d},
$$

where $\mu_{\lambda} \in \mathbb{R}$ is the associated Lagrange multiplier satisfying

$$
\mu_{\lambda}=\frac{1}{N}\left[e_{\lambda}(N)-\frac{1}{2} \int_{\mathbb{R}^{d}} \int_{\mathbb{R}^{d}} \frac{u_{\lambda}^{2}(x) u_{\lambda}^{2}(y)}{|x-y|^{2}} d x d y\right] .
$$

We next establish the following estimates.

Lemma 3.4. Suppose $g(x)$ satisfies $\left(\mathrm{M}_{1}\right)$ and $\left(\mathrm{M}_{2}\right)$ for $p>0$ and let $u_{\lambda}>0$ be a positive minimizer of $e_{\lambda}(N)$. Then we have

1. There exists a constant $C(p)>0$, depending only on $p>0$, such that the Lagrange multiplier $\mu_{\lambda}$ of (3.24) satisfies

$$
\mu_{\lambda} \leq C(p) \lambda^{\frac{2}{2+p}} \text { as } \lambda \rightarrow \infty \text {. }
$$

2. $u_{\lambda}$ has at least one maximal point $x_{\lambda}$, which satisfies

$$
u_{\lambda}\left(x_{\lambda}\right) \geq C_{0}(N, p) \lambda^{\frac{d}{2(2+p)}} \text { as } \lambda \rightarrow \infty
$$

for some constant $C_{0}(N, p)>0$.

Proof. (1) By Proposition 3.1 and Lemma 3.2, we deduce from (3.24) that

$$
\begin{aligned}
\mu_{\lambda} & =\frac{1}{N}\left[e_{\lambda}(N)-\frac{1}{2} \int_{\mathbb{R}^{d}} \int_{\mathbb{R}^{d}} \frac{u_{\lambda}^{2}(x) u_{\lambda}^{2}(y)}{|x-y|^{2}} d x d y\right] \\
& \leq \frac{1}{N} e_{\lambda}(N) \leq \frac{1}{N}\left[e_{\infty}(N)+o(1)\right] \lambda^{\frac{2}{2+p}} \leq C(p) \lambda^{\frac{2}{2+p}} \text { as } \lambda \rightarrow \infty,
\end{aligned}
$$

which then gives the estimate (3.25).

(2) Following (3.25) yields that $\liminf _{\lambda \rightarrow \infty}\left[\lambda g(x)-\mu_{\lambda}\right]>0$ for $|x|>R$, where $R>0$ is large enough. Together with (3.23), we then have

$$
-\Delta u_{\lambda}-\left(\int_{\mathbb{R}^{d}} \frac{u_{\lambda}^{2}(y)}{|x-y|^{2}} d y\right) u_{\lambda} \leq 0, \text { where }|x| \geq R>0 .
$$

The Hardy-Littlewood-Sobolev inequality (cf. Ref. 17, Theorem 4.3) gives that $\int_{\mathbb{R}^{d}} \frac{u_{\lambda}^{2}(y)}{|x-y|^{2}} d y \in L^{d}\left(\mathbb{R}^{d}\right)$. Following the De Giorgi-Nash-Moser theory (cf. Ref. 15, Theorem 4.1), we thus derive from (3.27) that for any $\xi \in B_{R}^{c}(0)$,

$$
\max _{x \in B_{1}(\xi)} u_{\lambda}(x) \leq C\left(\int_{B_{2}(\xi)} u_{\lambda}^{2}(x) d x\right)^{\frac{1}{2}}
$$


The above inequality implies that $u_{\lambda}(x)$ tends to zero near infinity and thus each $u_{\lambda}$ has at least one maximum point which we denote by $x_{\lambda}$.

We finally prove (3.26) as follows. For convenience, denote $\varepsilon_{\lambda}=\lambda^{-\frac{1}{2+p}}>0$. By contradiction, suppose the estimate (3.26) is false. Then for any small constant $\varepsilon>0$, we have

$$
u_{\lambda}\left(x_{\lambda}\right) \leq \varepsilon \varepsilon_{\lambda}^{-\frac{d}{2}} \text { in } \mathbb{R}^{d} .
$$

On the other hand, for any large $l>0$, it follows from Corollary 3.3 that

$$
\int_{\left\{g(x) \geq l \varepsilon_{\lambda}^{p}\right\}} u_{\lambda}^{2}(x) d x \leq \frac{1}{l \varepsilon_{\lambda}^{p}} \int_{\mathbb{R}^{d}} g(x) u_{\lambda}^{2}(x) d x \leq \frac{M_{2}}{l} .
$$

We thus deduce from (3.28) and (3.29) that for large $l>0$,

$$
\begin{aligned}
\frac{N}{2} \geq \frac{M_{2}}{l} & \geq \int_{\left\{g(x) \geq l \varepsilon_{\lambda}^{p}\right\}} u_{\lambda}^{2}(x) d x=N-\int_{\left\{g(x) \leq l \varepsilon_{\lambda}^{p}\right\}} u_{\lambda}^{2}(x) d x \\
& \geq N-\int_{\left\{|x| \leq 2 l^{\frac{1}{p}} \varepsilon_{\lambda}\right\}} u_{\lambda}^{2}(x) d x \geq N-\int_{\left\{|x| \leq 2 l^{\frac{1}{p}} \varepsilon_{\lambda}\right\}} \varepsilon^{2} \varepsilon_{\lambda}^{-d} d x \\
& \geq N-C \varepsilon^{2}>\frac{N}{2} \text { as } \lambda \rightarrow \infty .
\end{aligned}
$$

This is a contradiction, and hence (3.26) holds true. The proof is therefore complete.

In view of the estimate (3.10), we now define the $L^{2}\left(\mathbb{R}^{d}\right)$-normalized function as

$$
w_{\lambda}(x):=\varepsilon_{\lambda}^{\frac{d}{2}} u_{\lambda}\left(\varepsilon_{\lambda} x+x_{\lambda}\right), \varepsilon_{\lambda}:=\lambda^{-\frac{1}{2+p}}>0,
$$

where $x_{\lambda} \in \mathbb{R}^{d}$ is a maximal point of $u_{\lambda}$. We then deduce from (3.21) that

$$
\left\|\nabla w_{\lambda}\right\|_{2}^{2} \leq C(N, p),\left\|w_{\lambda}\right\|_{2}^{2}=N
$$

and note from (3.23) that $w_{\lambda}>0$ satisfies the following Euler-Lagrange equation:

$$
-\Delta w_{\lambda}(x)+\varepsilon_{\lambda}^{2} \lambda g\left(\varepsilon_{\lambda} x+x_{\lambda}\right) w_{\lambda}(x)=\varepsilon_{\lambda}^{2} \mu_{\lambda} w_{\lambda}(x)+\left(\int_{\mathbb{R}^{d}} \frac{w_{\lambda}^{2}(y)}{|x-y|^{2}} d y\right) w_{\lambda}(x) \text { in } \mathbb{R}^{d} .
$$

Lemma 3.5. Suppose $g(x)$ satisfies $\left(\mathrm{M}_{1}\right)$ and $\left(\mathrm{M}_{2}\right)$ for $p>0$ and let $u_{\lambda}>0$ be a positive minimizer of $e_{\lambda}(N)$. Then we have

1. The maximal point $x_{\lambda}$ of $u_{\lambda}$ satisfies

$$
\left|x_{\lambda}\right| \leq C(N, p) \varepsilon_{\lambda} \text { as } \lambda \rightarrow \infty,
$$

where $C(N, p)>0$ is independent of $\lambda$.

2. There exist sufficiently large $R=R(N, p)>0$ and $\lambda_{0}>0$ such that $w_{\lambda}$ satisfies

$$
w_{\lambda}(x) \leq C(N, p) e^{-|x|} \text { in } B_{R}^{c}(0) \text {, if } \lambda>\lambda_{0} .
$$

3. There exists a subsequence $\left\{w_{k}\right\}$ of $\left\{w_{\lambda_{k}}\right\}$, where $\lambda_{k} \rightarrow \infty$ as $k \rightarrow \infty$, and $0 \leq w_{0} \in H^{1}\left(\mathbb{R}^{d}\right)$ such that

$$
w_{k} \rightarrow w_{0} \text { in } H^{1}\left(\mathbb{R}^{d}\right) \text { as } k \rightarrow \infty
$$

and

$$
w_{k} \rightarrow w_{0} \text { strongly in } L^{q}\left(\mathbb{R}^{d}\right) \text { as } k \rightarrow \infty \text {, where, } 2 \leq q<2^{*} .
$$

Proof. (1) By contradiction, suppose (3.34) is false, which implies that

$$
\lim _{\lambda \rightarrow \infty}\left|x_{\lambda}\right| \varepsilon_{\lambda}^{-1} \rightarrow \infty, \text { where } \varepsilon_{\lambda}:=\lambda^{-\frac{1}{2+p}}>0 .
$$

Then we first claim that for any $x \in B_{2}(0)$,

$$
\varepsilon_{\lambda}^{-p} g\left(\varepsilon_{\lambda} x+x_{\lambda}\right) \rightarrow \infty \text { as } \lambda \rightarrow \infty .
$$


By the assumption $\left(\mathrm{M}_{1}\right)$, it is clear that the claim (3.39) is true for the case $x_{\lambda} \rightarrow 0$ as $\lambda \rightarrow \infty$. Therefore, the rest is to consider the case $x_{\lambda} \rightarrow 0$ as $\lambda \rightarrow \infty$. In fact, in the latter case, we obtain that $\left|\varepsilon_{\lambda} x+x_{\lambda}\right|$ is small in $B_{2}(0)$. By the assumption $\left(\mathrm{M}_{2}\right)$ for $p>0$, we derive from (3.38) that

$$
\varepsilon_{\lambda}^{-p} g\left(\varepsilon_{\lambda} x+x_{\lambda}\right) \geq \frac{1}{2} \varepsilon_{\lambda}^{-p}\left|\varepsilon_{\lambda} x+x_{\lambda}\right|^{p} \geq\left(\frac{1}{2}\right)^{p+1} \varepsilon_{\lambda}^{-p}\left|x_{\lambda}\right|^{p} \rightarrow \infty \text { as } \lambda \rightarrow \infty,
$$

where $x \in B_{2}(0)$. This establishes the claim (3.39).

We now observe from (3.25) that $\varepsilon_{\lambda}^{2} \mu_{\lambda} \leq C(p)$ as $\lambda \rightarrow \infty$. Together with (3.39), this estimate yields that $\varepsilon_{\lambda}^{-p} g\left(\varepsilon_{\lambda} x+x_{\lambda}\right)-\varepsilon_{\lambda}^{2} \mu_{\lambda}>0$ holds for large $\lambda>0$. Thus, we note from (3.33) that for large $\lambda>0$,

$$
-\Delta w_{\lambda}(x)-c_{\lambda}(x) w_{\lambda}(x) \leq 0 \text { in } B_{2}(0), \text { where } c_{\lambda}(x)=\int_{\mathbb{R}^{d}} \frac{w_{\lambda}^{2}(y)}{|x-y|^{2}} d y .
$$

By the Hardy-Littlewood-Sobolev inequality (cf. Ref. 17, Theorem 4.3), we have

$$
\left\|c_{\lambda}(x)\right\|_{L^{d}\left(B_{2}(0)\right)} \leq\left\|c_{\lambda}(x)\right\|_{L^{d}\left(\mathbb{R}^{d}\right)} \leq C\left\|w_{\lambda}(x)\right\|_{L^{\frac{2 d}{d-1}\left(\mathbb{R}^{d}\right)}}^{2} \leq C_{1}(N, p) \text { as } \lambda \rightarrow \infty .
$$

Using the De Giorgi-Nash-Moser theory (cf. Ref. 15, Theorem 4.1), we then obtain that

$$
w_{\lambda}(0) \leq C_{1}(N, p)\left(\int_{B_{2}(0)} w_{\lambda}^{2}(y) d y\right)^{\frac{1}{2}} \leq C_{2}(N, p) \text { as } \lambda \rightarrow \infty .
$$

On the other hand, by Hardy's inequality, we deduce from (3.32) that

$$
c_{\lambda}(0)=\int_{\mathbb{R}^{d}} \frac{w_{\lambda}^{2}(y)}{|y|^{2}} d y \leq C(N) \int_{\mathbb{R}^{d}}\left|\nabla w_{\lambda}\right|^{2} d y \leq C(N, p) .
$$

Since $w_{\lambda}(x)$ attains its maximum at $x=0$, we obtain from (3.33) and (3.43) that

$$
\varepsilon_{\lambda}^{-p} g\left(x_{\lambda}\right) w_{\lambda}(0) \leq \varepsilon_{\lambda}^{2} \mu_{\lambda} w_{\lambda}(0)+c_{\lambda}(0) w_{\lambda}(0) \leq \varepsilon_{\lambda}^{2} \mu_{\lambda} w_{\lambda}(0)+C(N, p) w_{\lambda}(0) .
$$

Recall from (3.26) that there exists some constant $C_{0}(N, p)>0$ such that

$$
w_{\lambda}(0) \geq C_{0}(N, p)>0 \text { as } \lambda \rightarrow \infty .
$$

By (3.42), (3.44), and (3.45), we thus conclude from (3.25) that

$$
C(p)+C(N, p) \geq \varepsilon_{\lambda}^{-p} g\left(x_{\lambda}\right) \text { as } \lambda \rightarrow \infty .
$$

Since it follows from (3.39) that $\varepsilon_{\lambda}^{-p} g\left(x_{\lambda}\right) \rightarrow \infty$ as $\lambda \rightarrow \infty$, we deduce a contradiction from the estimate (3.46). Therefore, the estimate (3.34) holds true.

(2) We note from (3.33) that $w_{\lambda}(x)$ satisfies

$$
-\Delta w_{\lambda}+\left[\varepsilon_{\lambda}^{-p} g\left(\varepsilon_{\lambda} x+x_{\lambda}\right)-\varepsilon_{\lambda}^{2} \mu_{\lambda}-c_{\lambda}(x)\right] w_{\lambda}=0 \text { in } \mathbb{R}^{d},
$$

where $c_{\lambda}(x)=\int_{\mathbb{R}^{d}} \frac{w_{\lambda}^{2}(y)}{|x-y|^{2}} d y$. If the estimates

$$
w_{\lambda}(x) \leq C(N, p) \quad \text { in } B_{R}^{c}(0)
$$

and

$$
\varepsilon_{\lambda}^{-p} g\left(\varepsilon_{\lambda} x+x_{\lambda}\right)-\varepsilon_{\lambda}^{2} \mu_{\lambda}-c_{\lambda}(x) \geq 1 \text { in } B_{R}^{c}(0)
$$

hold true for sufficiently large $R>0$ and $\lambda>0$, it then yields from the comparison principle that (3.35) holds; see Ref. 13 for the related argument. So, the rest is to establish (3.47) and (3.48).

By Lemma $3.4(1)$, we have $\varepsilon_{\lambda}^{2} \mu_{\lambda} \leq C(p)$ as $\lambda \rightarrow \infty$. We claim that for any $k>0$, there exists a sufficiently large constant $R=R(k)>0$ such that

$$
\varepsilon_{\lambda}^{-p} g\left(\varepsilon_{\lambda} x+x_{\lambda}\right)>k \quad \text { in } B_{R}^{c}(0),
$$

if $\lambda>0$ is large enough. In order to prove the claim (3.49), consider a small fixed constant $\varepsilon_{0}>0$. For any $k>0$, if $|x|>\frac{\varepsilon_{0}}{\varepsilon_{\lambda}}$, we then have

$$
\varepsilon_{\lambda}^{-p} g\left(\varepsilon_{\lambda} x+x_{\lambda}\right) \geq \varepsilon_{\lambda}^{-p} g\left(\frac{\varepsilon_{\lambda} x}{2}\right)>k \text { as } \lambda \rightarrow \infty
$$


and hence the estimate (3.49) is proved if $|x|>\frac{\varepsilon_{0}}{\varepsilon_{\lambda}}$. Next, if $R<|x| \leq \frac{\varepsilon_{0}}{\varepsilon_{\lambda}}$, we deduce that $\left|\varepsilon_{\lambda} x+x_{\lambda}\right|$ $\leq 2 \varepsilon_{0}$. This further implies from the assumption $\left(M_{2}\right)$ that $g\left(\varepsilon_{\lambda} x+x_{\lambda}\right) \geq \frac{1}{2}\left|\varepsilon_{\lambda} x+x_{\lambda}\right|^{p}$ for $R<|x| \leq \frac{\varepsilon_{0}}{\varepsilon_{\lambda}}$. Hence, for any $k>0$, take $R=\max \left\{2^{\frac{p+1}{p}} k^{\frac{1}{p}}, 2 C(N, p)\right\}>0$, where $C(N, p)>0$ is as in (3.34) so that for large $\lambda>0$,

$$
\left|\varepsilon_{\lambda} x+x_{\lambda}\right| \geq \frac{1}{2}\left|\varepsilon_{\lambda} x\right| \text { for } R<|x| \leq \frac{\varepsilon_{0}}{\varepsilon_{\lambda}}
$$

which then gives that for large $\lambda>0$,

$$
\begin{aligned}
\varepsilon_{\lambda}^{-p} g\left(\varepsilon_{\lambda} x+x_{\lambda}\right) & \geq \frac{1}{2} \varepsilon_{\lambda}^{-p}\left|\varepsilon_{\lambda} x+x_{\lambda}\right|^{p} \geq\left(\frac{1}{2}\right)^{p+1} \varepsilon_{\lambda}^{-p}\left|\varepsilon_{\lambda} x\right|^{p} \\
& >\left(\frac{1}{2}\right)^{p+1} R^{p}>k \text { for } R<|x| \leq \frac{\varepsilon_{0}}{\varepsilon_{\lambda}} .
\end{aligned}
$$

Therefore, the claim (3.49) is established.

Applying (3.25) and (3.49), we note that there exists a sufficiently large constant $R=R(N, p)>0$ such that for large $\lambda>0$,

$$
\varepsilon_{\lambda}^{-p} g\left(\varepsilon_{\lambda} x+x_{\lambda}\right)-\varepsilon_{\lambda}^{2} \mu_{\lambda} \geq 0 \text { in } B_{R}^{c}(0)
$$

and hence

$$
-\Delta w_{\lambda}(x)-c_{\lambda}(x) w_{\lambda}(x) \leq 0 \text { in } B_{R}^{c}(0), \text { where } c_{\lambda}(x)=\int_{\mathbb{R}^{d}} \frac{w_{\lambda}^{2}(y)}{|x-y|^{2}} d y
$$

if $R>0$ and $\lambda>0$ are large enough. Similar to proving (3.42), the estimate (3.47) then follows by applying the De Giorgi-Nash-Moser theory to (3.50). Therefore, the estimate (3.47) is now proved.

As for (3.48), we derive from (3.47) that for large $R>0$ and $\lambda>0$,

$$
\begin{aligned}
c_{\lambda}(x) & =\int_{\mathbb{R}^{d}} \frac{w_{\lambda}^{2}(y)}{|x-y|^{2}} d y=\int_{B_{1}(x)} \frac{w_{\lambda}^{2}(y)}{|x-y|^{2}} d y+\int_{B_{1}^{c}(x)} \frac{w_{\lambda}^{2}(y)}{|x-y|^{2}} d y \\
& \leq C(N, p) \int_{B_{1}(x)} \frac{1}{|x-y|^{2}} d y+\int_{B_{1}^{c}(x)} w_{\lambda}^{2}(y) d y \\
& \leq C(N, p) \text { in } B_{R}^{c}(0) .
\end{aligned}
$$

By taking sufficiently large $R=R(N, p)>0$, the estimate (3.48) therefore follows in view of (3.25), (3.49), and (3.51).

(3) Following (3.26) and (3.32), there exists a subsequence $\left\{w_{k}\right\}$ of $\left\{w_{\lambda_{k}}\right\}$, where $\lambda_{k} \rightarrow \infty$ as $k \rightarrow \infty$, and $0 \leq w_{0} \in H^{1}\left(\mathbb{R}^{d}\right)$ such that

$$
w_{k} \rightarrow w_{0} \neq 0 \text { in } H^{1}\left(\mathbb{R}^{d}\right) \text { as } k \rightarrow \infty
$$

and hence the estimate (3.36) holds true.

We next prove (3.37) as follows. Indeed, for any $l>0$, we have

$$
\int_{\left\{g(x) \geq l \varepsilon_{\lambda}^{p}\right\}} u_{\lambda}^{2}(x) d x \leq \frac{1}{l \varepsilon_{\lambda}^{p}} \int_{\mathbb{R}^{d}} g(x) u_{\lambda}^{2}(x) d x \leq \frac{M_{2}}{l} \text { as } \lambda \rightarrow \infty .
$$

We thus deduce from above that for any large $l>0$,

$$
\begin{aligned}
\frac{M_{2}}{l} & \geq \int_{\left\{g(x) \geq l \varepsilon_{\lambda}^{p}\right\}} u_{\lambda}^{2}(x) d x=N-\int_{\left\{g(x) \leq l \varepsilon_{\lambda}^{p}\right\}} u_{\lambda}^{2}(x) d x \\
& \geq N-\int_{\left\{|x| \leq 2 l^{\frac{1}{p}} \varepsilon_{\lambda}\right\}} u_{\lambda}^{2}(x) d x=N-\int_{\left\{\left|x-\frac{x_{\lambda}}{\varepsilon_{\lambda}}\right| \leq 2 l^{\frac{1}{p}}\right\}} w_{\lambda}^{2}(x) d x \\
& \geq N-\int_{\left\{|x| \leq 4 l^{\frac{1}{p}}\right\}} w_{\lambda}^{2}(x) d x \text { as } \lambda \rightarrow \infty,
\end{aligned}
$$

where (3.34) is used in the last inequality. By (3.52), we obtain that for any large $l>0$,

$$
w_{k} \rightarrow w_{0} \text { strongly in } L^{q}\left(\left\{|x| \leq 4 l^{\frac{1}{p}}\right\}\right) \text { as } k \rightarrow \infty,
$$

where $2 \leq q<2^{*}$. Therefore, we conclude from (3.54) and (3.55) that for any large $l>0$, 


$$
N \geq \int_{\left\{|x| \leq 4 l^{\frac{1}{p}}\right\}} w_{0}^{2}(x) d x \geq N-\frac{M_{2}}{l} .
$$

Since $l>0$ is arbitrary, we conclude from (3.56) that

$$
N=\left\|w_{0}\right\|_{2}^{2} \text { and } w_{k} \rightarrow w_{0} \text { strongly in } L^{2}\left(\mathbb{R}^{d}\right) \text { as } k \rightarrow \infty .
$$

Because $w_{k}$ is bounded uniformly in $H^{1}\left(\mathbb{R}^{d}\right)$, the estimate (3.37) is now proved. This completes the proof of Lemma 3.5.

Proof of Theorem 1.2. Suppose $u_{\lambda}$ is a positive minimizer of (1.4) and let $w_{\lambda}$ be defined by (3.31). Note from Lemma 3.5 that $w_{\lambda}$ satisfies the exponential decay (3.35) and there exists a subsequence $\left\{w_{k}\right\}$ of $\left\{w_{\lambda_{k}}\right\}$, where $\lambda_{k} \rightarrow \infty$ as $k \rightarrow \infty$, and $0 \leq w_{0} \in H^{1}\left(\mathbb{R}^{d}\right)$ such that $w_{k} \rightarrow w_{0}$ in $H^{1}\left(\mathbb{R}^{d}\right)$ and $w_{k} \rightarrow w_{0}$ strongly in $L^{q}\left(\mathbb{R}^{d}\right)$ as $k \rightarrow \infty$, where $2 \leq q<2^{*}$. It yields from (3.35) that $w_{0}(x) \leq C(N$, $p) e^{-|x|}$ in $B_{R}^{c}(0)$ for sufficiently large $R>0$. Applying (3.34) up to a subsequence of $\left\{\lambda_{k}\right\}$, we have $\lim _{k \rightarrow \infty} \frac{x_{\lambda_{k}}}{\varepsilon_{k}}=y_{0}$ for some $y_{0} \in \mathbb{R}^{d}$, where $x_{\lambda_{k}}$ is a maximal point of $u_{\lambda_{k}}$ and $\varepsilon_{k}=\lambda_{k}^{-\frac{1}{2+p}}>0$. Direct calculations now yield from (3.31) that

$$
\begin{aligned}
E_{\lambda_{k}}\left(u_{\lambda_{k}}\right)= & \int_{\mathbb{R}^{d}}\left|\nabla u_{\lambda_{k}}(x)\right|^{2} d x+\lambda_{k} \int_{\mathbb{R}^{d}} g(x) u_{\lambda_{k}}^{2}(x) d x-\frac{1}{2} \int_{\mathbb{R}^{d}} \int_{\mathbb{R}^{d}} \frac{u_{\lambda_{k}}^{2}(x) u_{\lambda_{k}}^{2}(y)}{|x-y|^{2}} d x d y \\
= & \varepsilon_{k}^{-2} \int_{\mathbb{R}^{d}}\left|\nabla w_{k}(x)\right|^{2} d x+\varepsilon_{k}^{-(2+p)} \int_{\mathbb{R}^{d}} g\left(\varepsilon_{k} x+x_{\lambda_{k}}\right) w_{k}^{2}(x) d x \\
& -\frac{1}{2} \varepsilon_{k}^{-2} \int_{\mathbb{R}^{d}} \int_{\mathbb{R}^{d}} \frac{w_{k}^{2}(x) w_{k}^{2}(y)}{|x-y|^{2}} d x d y \\
= & \varepsilon_{k}^{-2} \int_{\mathbb{R}^{d}}\left|\nabla w_{k}(x)\right|^{2} d x+\varepsilon_{k}^{-2} \int_{\mathbb{R}^{d}}\left|x+\frac{x_{\lambda_{k}}}{\varepsilon_{k}}\right|^{p} w_{k}^{2}(x) d x \\
& -\frac{1}{2} \varepsilon_{k}^{-2} \int_{\mathbb{R}^{d}} \int_{\mathbb{R}^{d}} \frac{w_{k}^{2}(x) w_{k}^{2}(y)}{|x-y|^{2}} d x d y \\
& +\varepsilon_{k}^{-2} \int_{\mathbb{R}^{d}}\left(\varepsilon_{k}^{-p} g\left(\varepsilon_{k} x+x_{\lambda_{k}}\right)-\left|x+\frac{x_{k}}{\varepsilon_{k}}\right|^{p}\right) w_{k}^{2}(x) d x .
\end{aligned}
$$

Similar to (3.12), one can prove that

$$
\int_{\mathbb{R}^{d}}\left(\varepsilon_{k}^{-p} g\left(\varepsilon_{k} x+x_{\lambda_{k}}\right)-\left|x+\frac{x_{k}}{\varepsilon_{k}}\right|^{p}\right) w_{k}^{2}(x) d x=o(1) \text { as } k \rightarrow \infty .
$$

We then deduce from above that

$$
\begin{aligned}
& \liminf _{k \rightarrow \infty} \lambda_{k}{ }^{-\frac{2}{2+p}} e_{\lambda_{k}}(N)=\liminf _{k \rightarrow \infty} \varepsilon_{k}^{2} E_{\lambda_{k}}\left(u_{\lambda_{k}}\right) \\
\geq & \int_{\mathbb{R}^{d}}\left|\nabla w_{0}(x)\right|^{2} d x+\int_{\mathbb{R}^{d}}\left|x+y_{0}\right|^{p} w_{0}^{2}(x) d x-\frac{1}{2} \int_{\mathbb{R}^{d}} \int_{\mathbb{R}^{d}} \frac{w_{0}^{2}(x) w_{0}^{2}(y)}{|x-y|^{2}} d x d y \\
= & \int_{\mathbb{R}^{d}}\left|\nabla \hat{w}_{0}(x)\right|^{2} d x+\int_{\mathbb{R}^{d}}|x|^{p} \hat{w}_{0}^{2}(x) d x-\frac{1}{2} \int_{\mathbb{R}^{d}} \int_{\mathbb{R}^{d}} \frac{\hat{w}_{0}^{2}(x) \hat{w}_{0}^{2}(y)}{|x-y|^{2}} d x d y \geq e_{\infty}(N),
\end{aligned}
$$

where $\hat{w}_{0}(x)=w_{0}\left(x-y_{0}\right) \geq 0$. Following Lemma 3.2, the above inequalities must be identities, which further imply that $\hat{w}_{0} \geq 0$ is a minimizer of $e_{\infty}(N)$. Therefore,

$$
e_{\infty}(N)=E_{\infty}\left(\hat{w}_{0}(x)\right), \quad 0<N<N^{*}
$$

and

$$
\lim _{k \rightarrow \infty}\left\|\nabla w_{k}(x)\right\|_{2}^{2}=\left\|\nabla w_{0}(x)\right\|_{2}^{2} \text { and } w_{k}(x) \rightarrow w_{0}(x)=\hat{w}_{0}\left(x+y_{0}\right) \text { in } H^{1}\left(\mathbb{R}^{d}\right) \text { as } k \rightarrow \infty,
$$

which implies that the limit behavior (1.17) now follows from (3.31) and (3.59). Also, $\hat{w}_{0} \geq 0$ satisfies the Euler-Lagrange equation (1.18) for some Lagrange multiplier $\mu=\mu(N) \in \mathbb{R}$ such that $\int_{\mathbb{R}^{d}} \hat{w}_{0}^{2}=N$. Moreover, the maximum principle applied to (1.18) yields that $\hat{w}_{0}>0$ in $\mathbb{R}^{d}$. Similar to Ref. 13, one can further derive that $\hat{w}_{0}>0$ is essentially a ground state of (1.18). 
The rest is to prove that $y_{0}=0$. On the contrary, suppose that $\left|y_{0}\right|>0$. Since $w_{k}(0)=\max _{x \in \mathbb{R}^{d}} w_{k}(x)$ for all $k>0$, we deduce from (3.59) that $\hat{w}_{0}\left(\left|y_{0}\right|\right)=\max _{x \in \mathbb{R}^{d}} \hat{w}_{0}(x)$, which is a contradiction to the fact that $\hat{w}_{0}>0$ is strictly decreasing in $|x|$ from Proposition 3.1. Therefore, we have $y_{0}=0$, which then completes the proof of Theorem 1.2.

\section{ACKNOWLEDGMENTS}

The authors would like to thank the referee for helpful comments and suggestions on our manuscript. The first author thanks Professor Robert Seiringer for introducing him the related interesting phenomena addressed in this paper and for constant encouragements over the past few years. Part of this paper was finished when the first author visited the Pacific Institute for Mathematical Sciences (PIMS) at UBC from January to February in 2018. He would like to thank PIMS for their warm hospitality. Y. J. Guo is partially supported by NSFC under Grant No. 11671394 and MOST under Grant No. 2017YFA0304500. Z.-Q. Wang is partially supported by NSFC under Grant No. 11771324.

${ }^{1}$ Bartsch, T., Pankov, A., and Wang, Z.-Q., "Nonlinear Schrödinger equations with steep potential well," Commun. Contemp. Math. 3, 549-569 (2001).

${ }^{2}$ Byeon, J. and Wang, Z.-Q., "Standing waves with a critical frequency for nonlinear Schrödinger equations," Arch. Ration. Mech. Anal. 165, 295-316 (2002).

${ }^{3}$ Caffarelli, L., Kohn, R., and Nirenberg, L., "First order interpolation inequalities with weights," Compositio Math. 53, 259-275 (1984)

${ }^{4}$ Cao, D. M. and Su, Y. M., "Minimal blow-up solutions of mass-critical inhomogeneous Hartee equation,” J. Math. Phys. 54, 121511 (2013).

${ }^{5}$ Cao, P., Wang, J., and Zou, W. M., "On the standing waves for nonlinear Hartree equation with confining potential," J. Math. Phys. 53, 033702 (2012).

${ }^{6}$ Cazenave, T., Semilinear Schrödinger Equations, Courant Lecture Notes in Mathematics Vol. 10 (Courant Institute of Mathematical Science/AMS, New York, 2003).

${ }^{7}$ Cazenave, T. and Lions, P. L., "Orbital stability of standing waves for some nonlinear Schrödinger equations," Commun. Math. Phys. 85, 549-561 (1982).

${ }^{8}$ Deng, Y. B., Guo, Y. J., and Lu, L., "On the collapse and concentration of Bose-Einstein condensates with inhomogeneous attractive interactions," Calculus Var. Partial Differ. Equations 54, 99-118 (2015).

${ }^{9}$ Deng, Y. B., Lin, C. S., and Yan, S., "On the prescribed scalar curvature problem in $\mathbb{R}^{N}$, local uniqueness and periodicity," J. Math. Pures Appl. 104, 1013-1044 (2015).

${ }^{10}$ Deng, Y. B., Lu, L., and Shuai, W., "Constraint minimizers of mass critical Hartree energy functionals: Existence and mass concentration,” J. Math. Phys. 56, 061503 (2015).

${ }^{11}$ Gilbarg, D. and Trudinger, N. S., Elliptic Partial Differential Equations of Second Order, Grundlehren der Mathematischen Wissenschaften Vol. 224 (Springer, Berlin, 1983).

${ }^{12}$ Guo, Y. J. and Seiringer, R., "On the mass concentration for Bose-Einstein condensates with attractive interactions," Lett. Math. Phys. 104, 141-156 (2014).

${ }^{13}$ Guo, Y. J., Wang, Z.-Q., Zeng, X. Y., and Zhou, H. S., "Properties for ground states of attractive Gross-Pitaevskii equations with multi-well potentials," Nonlinearity 31, 957-979 (2018).

${ }^{14}$ Guo, Y. J., Zeng, X. Y., and Zhou, H. S., "Energy estimates and symmetry breaking in attractive Bose-Einstein condensates with ring-shaped potentials," Ann. Inst. Henri Poincare (C) Non Linear Anal. 33(3), 809-828 (2016).

${ }^{15}$ Han, Q. and Lin, F. H., Elliptic Partial Differential Equations, 2nd ed., Courant Lecture Notes in Mathematics Vol. 1 (Courant Institute of Mathematical Science/AMS, New York, 2011).

${ }^{16}$ Lieb, E. H., "Existence and uniqueness of the minimizing solution of Choquard's nonlinear equation," Stud. Appl. Math. 57, 93-105 (1977).

${ }^{17}$ Lieb, E. H. and Loss, M., Analysis, 2nd ed., Graduate Studies in Mathematics Vol. 14 (American Mathematical Society, Providence, RI, 2001).

${ }^{18}$ Lions, P. L., "The Choquard equation and related questions,” Nonlinear Anal. 4, 1063-1072 (1980).

${ }^{19}$ Lions, P. L., "The concentration-compactness principle in the calculus of variations. The locally compact case, part 1," Ann. Inst. Henri Poincare (C) Non Linear Anal. 1, 109-145 (1984); "The concentration-compactness principle in the calculus of variations. The locally compact case, part 2," 1, 223-283 (1984).

${ }^{20}$ Luo, Y., "Uniqueness of ground states for nonlinear Hartree equations" (submitted).

${ }^{21}$ Ma, L. and Zhao, L., "Classification of positive solitary solutions of the nonlinear Choquard equation," Arch. Ration. Mech. Anal. 195, 455-467 (2010).

22 Moroz, V. and Van Schaftingen, J., "Semi-classical states for the Choquard equation," Calculus Var. Partial Differ. Equations 52, 199-235 (2015).

${ }^{23}$ Moroz, V. and Van Schaftingen, J., "Ground states of nonlinear Choquard equation: Existence, qualitative properties and decay asymptotics," J. Funct. Anal. 265, 153-184 (2013).

${ }^{24}$ Willem, M., Minimax Theorems, Progress in Nonlinear Differential Equations and Their Applications Vol. 24 (Birkhäuser Boston, Inc., Boston, 1996). 